\title{
Transcranial alternating current stimulation (tACS): from basic mechanisms towards first applications in psychiatry
}

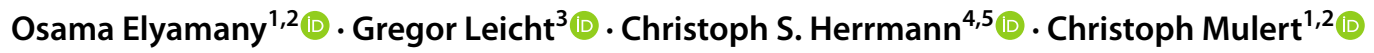

Received: 25 March 2020 / Accepted: 27 October 2020 / Published online: 19 November 2020

(c) The Author(s) 2020

\begin{abstract}
Transcranial alternating current stimulation (tACS) is a unique form of non-invasive brain stimulation. Sinusoidal alternating electric currents are delivered to the scalp to affect mostly cortical neurons. tACS is supposed to modulate brain function and, in turn, cognitive processes by entraining brain oscillations and inducing long-term synaptic plasticity. Therefore, tACS has been investigated in cognitive neuroscience, but only recently, it has been also introduced in psychiatric clinical trials. This review describes current concepts and first findings of applying tACS as a potential therapeutic tool in the field of psychiatry. The current understanding of its mechanisms of action is explained, bridging cellular neuronal activity and the brain network mechanism. Revisiting the relevance of altered brain oscillations found in six major psychiatric disorders, putative targets for the management of mental disorders using tACS are discussed. A systematic literature search on PubMed was conducted to report findings of the clinical studies applying tACS in patients with psychiatric conditions. In conclusion, the initial results may support the feasibility of tACS in clinical psychiatric populations without serious adverse events. Moreover, these results showed the ability of tACS to reset disturbed brain oscillations, and thus to improve behavioural outcomes. In addition to its potential therapeutic role, the reactivity of the brain circuits to tACS could serve as a possible tool to determine the diagnosis, classification or prognosis of psychiatric disorders. Future double-blind randomised controlled trials are necessary to answer currently unresolved questions. They may aim to detect response predictors and control for various confounding factors.
\end{abstract}

Keywords Transcranial alternating current stimulation (tACS) · Non-invasive brain stimulation (NIBS) · Psychiatry · Schizophrenia · Depression · OCD

\section{Abbreviations}

$\mathrm{AD}$

Alzheimer's disease

ADAS-Cog The 11-item cognitive subscale of the Alzheimer's Disease Assessment Scale

ADHD Attention deficit hyperactivity disorder,

Christoph Mulert

Christoph.Mulert@psychiat.med.uni-giessen.de

1 Centre of Psychiatry, Justus-Liebig University, Klinikstrasse 36, 35392 Giessen, Hessen, Germany

2 Centre for Mind, Brain and Behaviour (CMBB), University of Marburg and Justus-Liebig University Giessen, Marburg, Germany

3 Department of Psychiatry and Psychotherapy, University Medical Centre Hamburg-Eppendorf, Hamburg, Germany

4 Experimental Psychology Lab, Centre for Excellence "Hearing4all," European Medical School, University of Oldenburg, Oldenburg, Lower Saxony, Germany

5 Research Centre Neurosensory Science, University of Oldenburg, Oldenburg, Lower Saxony, Germany
AHRS

ASSR

BACS

BDI

CES

CGI

DLPFC

DMPFC

DTI

EEG

FAA

HAMA

hdEEG

HDRS

LTD

LTP

M1

MADRS
Auditory Hallucination Rating Scale

Auditory steady-state response

Brief assessment of cognition in

schizophrenia

Beck Depression Inventory

Cranial electrotherapy stimulation

Clinical Global Impression Scale

Dorsolateral prefrontal cortex

Dorsomedial prefrontal cortex

Diffusion tensor imaging

Electroencephalography

Frontal alpha asymmetry

Hamilton Anxiety Rating Scale

High-density electroencephalography

Hamilton Depression Rating Scale

Long-term-depression

Long-term-potentiation

Primary motor

Montgomery-Asberg Depression Rating

Scale 


$\begin{array}{ll}\text { MCI } & \text { Mild cognitive impairment } \\ \text { MDD } & \text { Major depressive disorder } \\ \text { MEG } & \text { Magnetoencephalography } \\ \text { NIBS } & \text { Non-invasive brain stimulation } \\ \text { OCD } & \text { Obsessive-compulsive disorder } \\ \text { PANAS } & \text { Positive and Negative Affect Schedule } \\ \text { PANSS } & \text { Positive and Negative Syndrome Scale } \\ \text { PMA } & \text { Premotor area } \\ \text { PSYRATS } & \text { Psychiatry rating scale } \\ \text { SANS } & \text { Scale for the Assessment of Negative } \\ & \text { Symptoms } \\ \text { SAPS } & \text { Scale for the Assessment of Positive } \\ & \text { Symptoms } \\ \text { SMA } & \text { Supplementary motor area } \\ \text { STDP } & \text { Spike-timing-dependent plasticity } \\ \text { SUMD } & \text { Scale to Assess Unawareness of Mental } \\ & \text { Disorder } \\ \text { tACS } & \text { Transcranial alternating current stimulation } \\ \text { tDCS } & \text { Transcranial direct current stimulation } \\ \text { TMS } & \text { Transcranial magnetic stimulation } \\ \text { TMT } & \text { Trail Making Test } \\ \text { WM } & \text { Working memory } \\ \text { YBOCS } & \text { Yale-Brown Obsessive-compulsive Scale }\end{array}$

\section{Introduction}

Transcranial alternating current stimulation (tACS) is a widely used non-invasive brain stimulation (NIBS) method. It has been used for more than a decade in different fields, such as cognitive neuroscience [1,2]. However, its use in psychiatric clinical research began with case reports [3], and only recently have the first well-structured double-blind randomized controlled trials examined its efficacy for the treatment of psychiatric disorders [4]. tACS involves direct delivery of alternating electric currents to the scalp. The current travels through the skull to affect mostly cortical neurons. Such alternating current has a sinusoidal waveform where the voltage changes gradually from positive to negative every half-cycle. Therefore, the current flows from an anodal electrode to a cathodal electrode in one half-cycle and in the reverse direction in the second half-cycle [5].

The concept underlying alternating current is to simulate the naturally occurring rhythmic pattern of electrophysiological activity of the brain, which can be detected by electroencephalography (EEG) and magnetoencephalography (MEG) [6]. Such rhythmic patterns, oscillating at a certain frequency, are called brain oscillations. Various specific brain oscillations have been coupled with diverse brain functions and states [7]. Moreover, connectivity and communication between distant cortical regions were shown to be associated with the synchronization of brain oscillations within these regions $[8,9]$. In that sense, tACS is also used to couple or decouple two connected neuronal circuits by synchronizing or desynchronizing their oscillations, respectively. Accordingly, tACS might represent a potential therapeutic tool by modifying altered brain oscillations and connectivity patterns that were previously identified in various psychiatric disorders.

The typical setup of tACS involves the application of electrodes onto the scalp, whose position and size can be modified to specifically target a certain brain region $[10,11]$. For this purpose, positioning of the electrodes is designed according to computational models to optimize the stimulation parameters $[12,13]$. Furthermore, the parameters of the alternating current itself can be customized in terms of frequency, amplitude, phase shape, phase timing, and the duration and number of stimulation sessions. The stimulation frequency is usually set to EEG frequencies to modulate the brain processes associated with them. Other parameters may vary according to the study question and brain electric activity to be modulated (some examples are discussed below; for more details, see this review: [14]).

\section{Mechanism of action}

Although the exact mechanisms of action of tACS are still not well understood, there is growing evidence of possible explanations. Electrophysiological methods have been extensively used to elucidate these mechanisms, as well as the effects of tACS, mostly EEG and, to a lesser degree, MEG, in humans, and intracranial and local field potential recordings in animals $[15,16]$. Those methods have shown two main categories of tACS effects: online effects (those that coincide with the stimulation duration), and offline effects or after-effects (those that outlast the stimulation period). Both involve entrainment of brain oscillations to the stimulation frequency and coupling or decoupling of long-range oscillatory connectivity between distant brain regions [17, 18]. To understand these effects and their underlying electrophysiological processes, we summarize previous literature bridging the gap between cellular and whole network levels.

Upon tACS application to the skull, some of this alternating current reaches the brain. As a result, it causes the cell bodies and dendrites of the cortical neurons to alter their membrane potential towards depolarization or hyperpolarization in an oscillatory fashion [16]. This alternating change in the membrane potential is thought to be sufficient to alter the probability of a neuron generating action potentials [19]. However, it is not strong enough to change the rate of action potentials, as it controls only their timing in a frequency- and location-specific manner [20]. That is why this stimulation seems to be a type of sub-threshold stimulation that does not directly fire neuronal spikes $[14,21]$. 
The influence of tACS depends not only on the amplitude and frequency but also on the three-dimensional orientation of both neurons and the penetrating current [22]. Its effects result from manipulating the membrane potential of neurons that are aligned with the introduced electric field, mostly pyramidal cells in layer V. These cells are extremely sensitive to current changes due to their elongated somadendritic axis [22, 23]. Moreover, they have characteristic properties, including intrinsic resonance, neuroplastic activity and cortico-cortical projections [24-26]. Therefore, these cells, once stimulated by tACS, show specific frequency resonance, long-term after-effects and long-range oscillatory cortical connectivity (more details on resonance and aftereffects are discussed below). Similarly, the direction of the electric stimulation substantially changes the properties of the resulting field, and thus, its effects on the neurons [27].

In 2018, Liu et al. suggested five different neuronal mechanisms that could translate the previously mentioned cellular effects of tACS into whole network activity at a larger scale [28]. First, stochastic resonance: a wide range of tACSaffected neurons, differing in their momentary probability to generate action potentials, will react stochastically to be either polarized or hyperpolarized. This leads to the absence of a theoretical 'minimum effective threshold'. Second, rhythm resonance: this occurs when the tACS frequency is the same as that of the endogenous oscillations. This ends with the stimulatory current wave striking the endogenous one at a similar phase every cycle. Third, temporal biasing of spikes: the spike timing of neurons is regulated by the interaction between the stimulation and internal currents, which may work synergistically to excite the same group of neurons during each cycle of stimulation. Fourth, network entrainment: the entrainment of an endogenous irregular activity necessitates an external current with sufficiently stronger amplitude. Finally, imposed pattern: to overcome endogenous regular oscillations and introduce a new oscillation, the strongest stimulation is required [28].

These mechanisms support the explanation by Vosskuhl et al. [14] of the large-scale effects of tACS. The authors attributed the online and offline tACS effects to two synergistic phenomena: entrainment and neuroplasticity, respectively. Entrainment, by definition, takes place when an external rhythmic system affects another naturally occurring one, forcing it to follow its own oscillating frequency. During tACS, the external driving current forces the endogenous brain oscillations to follow in terms of frequency and phase $[14,29]$. Such entrainment has two crucial properties: "Arnold tongue", and harmonics. Arnold tongues describe the relationship between the stimulation amplitude and its corresponding range of frequencies at which tACS can entrain endogenous brain oscillations. When the amplitude of this stimulation increases, it entrains brain oscillations in a wider range of frequencies [30]. Harmonics describe the preference of an intrinsic oscillator to be trained by tACS at multiple frequencies that have an $\mathrm{n}: \mathrm{m}$ relationship to the endogenous frequency, e.g., at twice or half the endogenous frequency $[23,31]$.

For the neuroplasticity to elicit offline effects, long-term plasticity should occur in one form among two primary mechanisms: Long-term-potentiation (LTP) and long-termdepression (LTD), two results of spike-timing-dependent plasticity (STDP) [32]. LTP is the potentiation of a synaptic connection when the presynaptic action potential comes before the post-synaptic potential. In contrast, LTD is the weakening of the synapse if the presynaptic action potential follows the post-synaptic one. Therefore, these phenomena are the primary culprits that elicit offline tACS effects by increasing or decreasing neural synchronization [33]. This explanation has been confirmed by many studies [33-36], which may explain why the offline effects of tACS have been shown to last for $70 \mathrm{~min}$ after one stimulation session lasting $20 \mathrm{~min}$ [37].

From this standpoint, we try to refer to some unique features of tACS as a neuromodulator in contrast to other forms of NIBS, yet it is relatively new and the least studied form. Transcranial direct current stimulation (tDCS), a closely related type of NIBS that constantly depolarizes or hyperpolarizes the affected neurons [38], specifically changing axonal membrane potential [39]. In fact, tACS is a specific version of tDCS where the current is set to fluctuate sinusoidally between the electrodes rather than exhibiting constant polarity. Similar to tACS, tDCS effects depend on the orientation of the neurons relative to the current direction [40, 41], and it can induce both online and offline neuroplastic effects [42, 43]. Equivalently, tDCS does not directly affect the neuronal firing rate, but rather its probability and spontaneous activity via subthreshold voltage changes [44]. Although tDCS is shown to modulate oscillatory brain activity [45], tACS is more effective at entraining the endogenous brain oscillations as it mimics the alternating nature of brain oscillations [2, 14, 46] (For more details about the mechanisms of action of tDCS, see these reviews: [47-51]).

Another more studied method of NIBS is transcranial magnetic stimulation (TMS), which is approved for the treatment of some psychiatric disorders [52-54]. In TMS, a magnetic field is produced by a coil applied to the scalp and then travels through the skull to elicit electric fields in the cortical neurons [55]. Compared to tACS, TMS, a suprathreshold stimulator, produces action potentials in silent neurons [56] in the form of two successive volleys. While the first volley (direct waves) represents direct activation of pyramid tract axons, the second (indirect waves) reflects synaptic activation of the same neurons [57]. As expected, TMS evokes long-term synaptic changes and thus after-effects beyond the stimulation period [58] (For more details about the mechanisms of action of TMS, see 
these reviews: [59-63]). However, practically, tACS exhibits superior cost, portability, tolerability, and safety profiles [64, 65]. In other words, tACS is a feasible tool that reshapes or re-synchronizes intrinsic brain rhythms, manipulating the associated brain functions without adding extra excitatory or inhibitory burden. Given that (1) tACS clinical research is still in its infancy, and (2) tACS possesses such unique features, we aim, in this review, to encourage more tACS usage in psychiatric research.

\section{Altered brain oscillations and tACS applications in psychiatric disorders:}

Given the association between EEG brain oscillations and various brain functions, many researchers have managed to successfully modulate normal cognitive functions by manipulating brain oscillations or connectivity patterns using tACS (for more details see these reviews [7, 14, 65-67]). Motivated by successful tACS applications in cognition, future investigations could aim to normalize pathological brain oscillations, and identify beneficial tACS roles in the management of psychiatric disorders. In this section, we focus on such electrophysiological alterations that could benefit researchers in designing tACS clinical trials in psychiatric patients. Moreover, we review previous clinical trials that have already examined the role of tACS in psychiatry. Six psychiatric disorders are discussed, where a subsection is devoted to each disorder.

It is noteworthy to state two caveats here to properly understand the current state of the literature regarding disturbed brain oscillations in psychiatry. First, this is not a comprehensive overview of disturbed brain oscillations in all psychiatric disorders, but rather, it presents six of the most studied and major disorders. Hence, this section is intended to provide only a glimpse of this interesting electrophysiological approach in psychiatric pathophysiology. Second, because of the heterogeneity of the studies and the limited knowledge of some disturbed oscillations, we tried to selectively focus on some of the more replicated and reproduced findings supported by different studies. Therefore, the following electrophysiological changes should be extrapolated with extreme caution before building around them to design tACS protocols.

The results of tACS clinical studies were identified according to a systematic search on PubMed using two keywords with the Boolean operator "AND". The first keyword was "tACS" or "alternating current stimulation" throughout the search process. The second term was changeable to signify several psychiatric disorders ("ADHD", "Insomnia", "Depression", "Schizophrenia", "Bipolar", "OCD”, "Anxiety", "PTSD", "Dementia", and "Alzheimer"). The search process, last performed in June 2020, yielded a total of 151 records, including 68 duplicates. The original 83 publications were screened to exclude 31 non-tACS relevant articles and 15 reviews. After a careful assessment of the remaining 37 studies, a stroke-related article and 18 non-clinical experiments were excluded. Five publications actually applied cranial electrotherapy stimulation (CES) rather than tACS, and thus were excluded. The remaining 13 eligible articles, which show the experimental application of tACS in patients with any psychiatric disorder, were included (see Fig. 1 [68]).

The included articles comprise study designs with different levels of evidence: three randomized double-blind controlled trials, two single-blind randomized controlled trials, an open-label non-controlled clinical trial, a longitudinal case-control study, two case series, and four case reports (see Table 1). They cover five different psychiatric disorders: one study on attention-deficit/hyperactivity disorder (ADHD), three publications on depression, seven on schizophrenia, one on dementia, and one case series on OCD. Some of the included articles conducted a relatively non-robust study design, such as case reports. However, we will consider them in further discussion due to the limited number of clinical trials and the variety of investigated psychiatric disorders. Accordingly, the reader could appreciate the shortage of evidence to apply tACS in psychiatry. After discussing the included articles for each disorder, we state some general remarks on the application of tACS in participants with psychiatric illnesses.

\section{Schizophrenia}

With the aid of EEG and MEG, a great bulk of research has already identified characteristic alterations of brain oscillations in patients with schizophrenia [69]. These electrophysiological fingerprints of schizophrenia have been shown to be heterogeneous but task-/state-, location, and frequencyspecific, where they, in turn, correlate with the severity of certain symptoms [70]. However, such heterogeneity is not surprising given the heterogeneity of clinical presentations in schizophrenia [71]. Pertaining to the task/state aspect, three distinctive patterns of brain oscillations were identified: evoked, induced and resting-state [69]. Each type could be compromised peculiarly in schizophrenia, so tACS strategies should precisely consider which one/ones to modify.

Regarding the frequency and location domains, the results might be harmonious in the low-frequency range (alpha and theta), whereas they are not in the high-frequency range (beta and gamma). A general persistent finding in schizophrenia is reduced alpha power, especially in the resting-state, which might be linked to the increased state of arousal and abnormal self-referential processing [70, 72-76]. Delta and theta waves are mostly elevated [77-79], but only theta activity is decreased, especially in the frontal lobe [80], correlating 

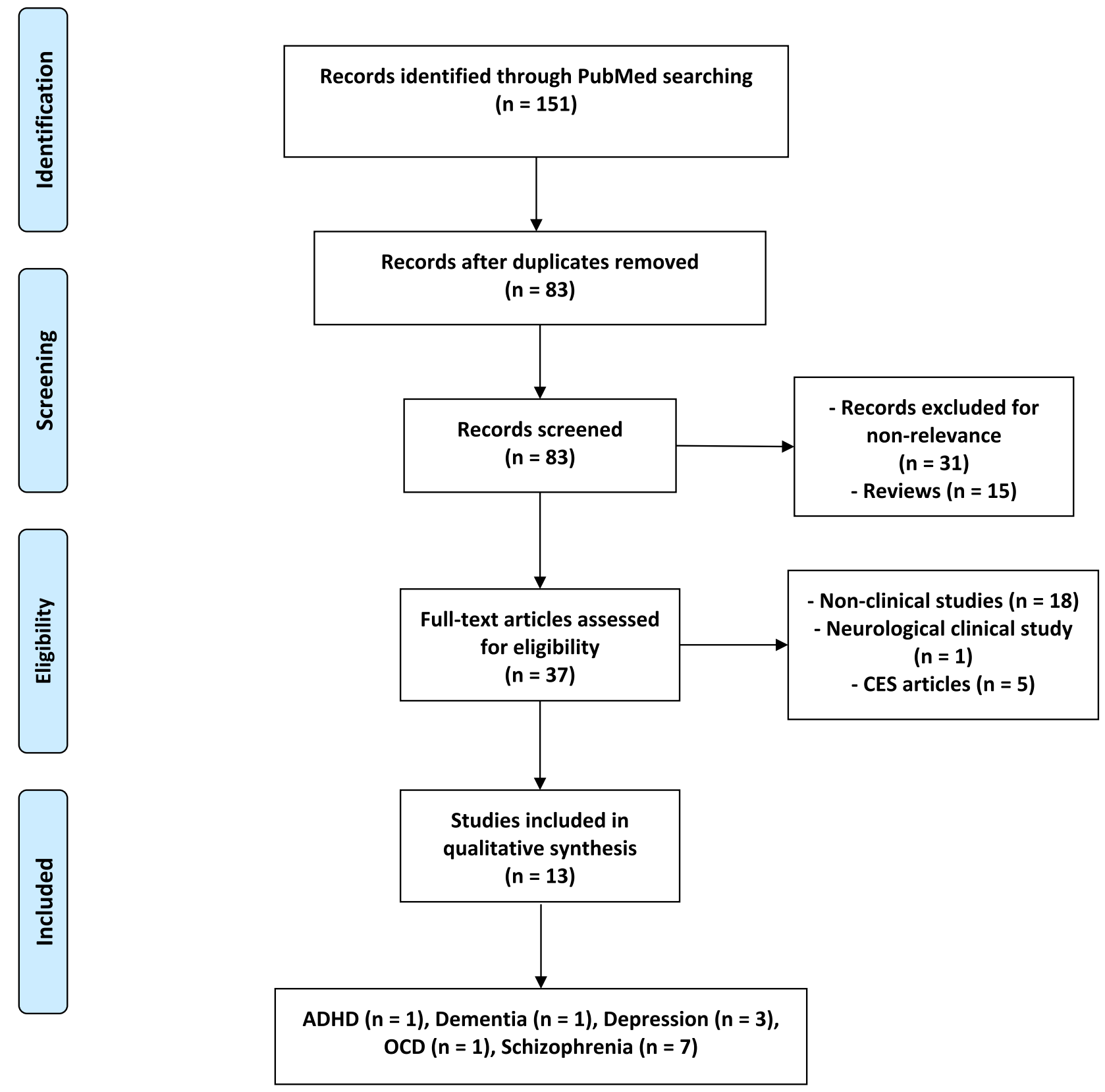

Fig. 1 PRISMA Flow Diagram of the included articles [68]. ADHD attention deficit hyperactivity disorder, CES cranial electrotherapy stimulation, $O C D$ obsessive-compulsive disorder

with an impairment in working memory [81, 82]. Furthermore, theta-band connectivity was shown to be increased, especially between the frontal and parietal regions, during the resting state $[83,84]$. Although beta activity in schizophrenia is not well-studied, some studies showed that it was decreased in both resting-state and task-related brain activity $[85,86]$. Alterations of gamma-band oscillations have been extensively investigated as a neurobiological correlate of different stages and symptoms of schizophrenia [87] and are commonly interpreted as an imbalance between excitation and inhibition (E/I-balance), which is considered a crucial mechanism in the pathophysiology of schizophrenia [88].

A major and often replicated finding in schizophrenia is the reduction of stimulus-evoked task-related gamma power and coherence between different brain regions [85, 89-95], which, besides the chronic illness, appears across different stages and models of the disease, including in patients with their first episode of psychosis $[95,96]$, in healthy relatives of patients [97, 98], in subjects at high risk for developing psychosis [99], and in the ketamine model of schizophrenia 


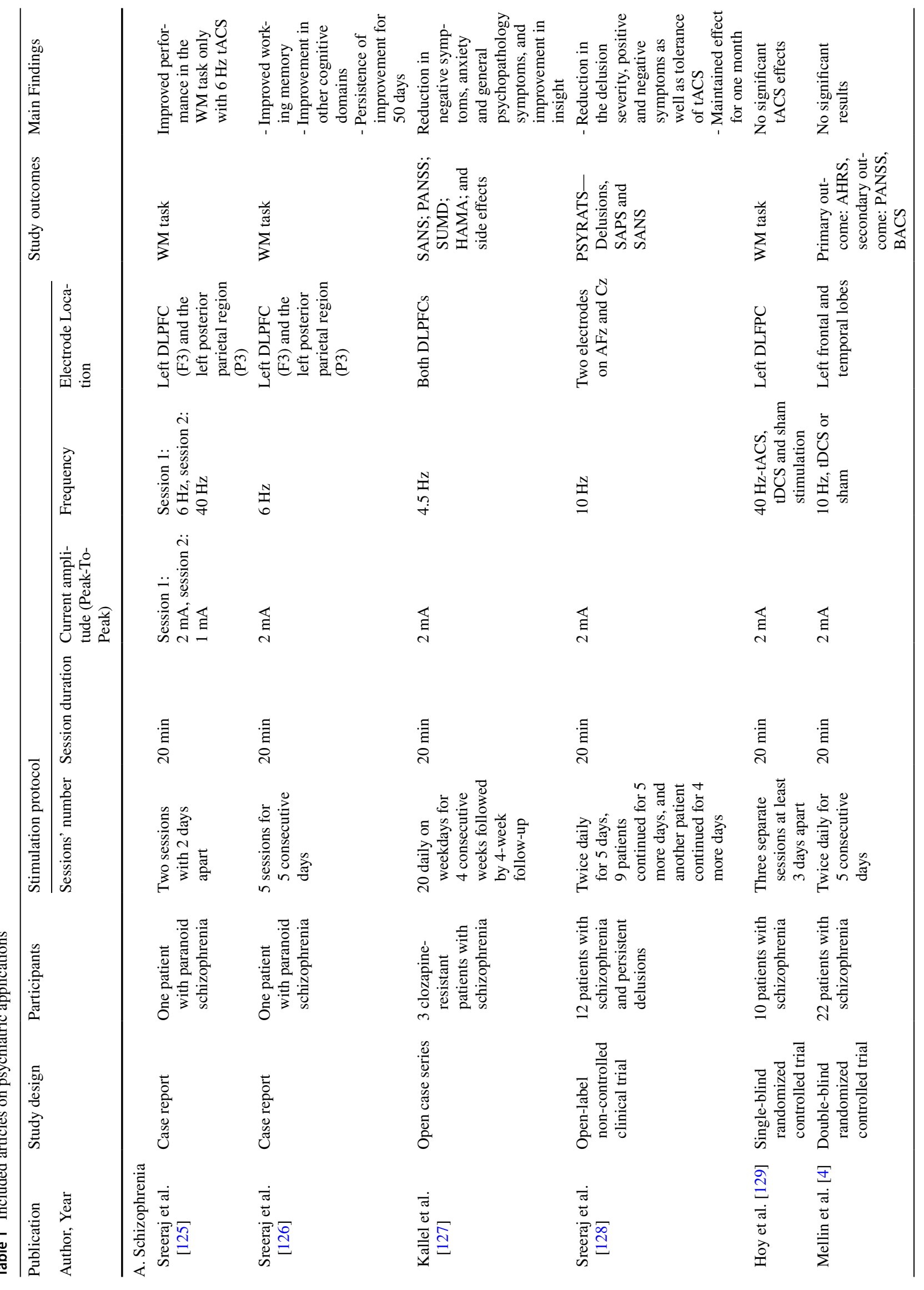




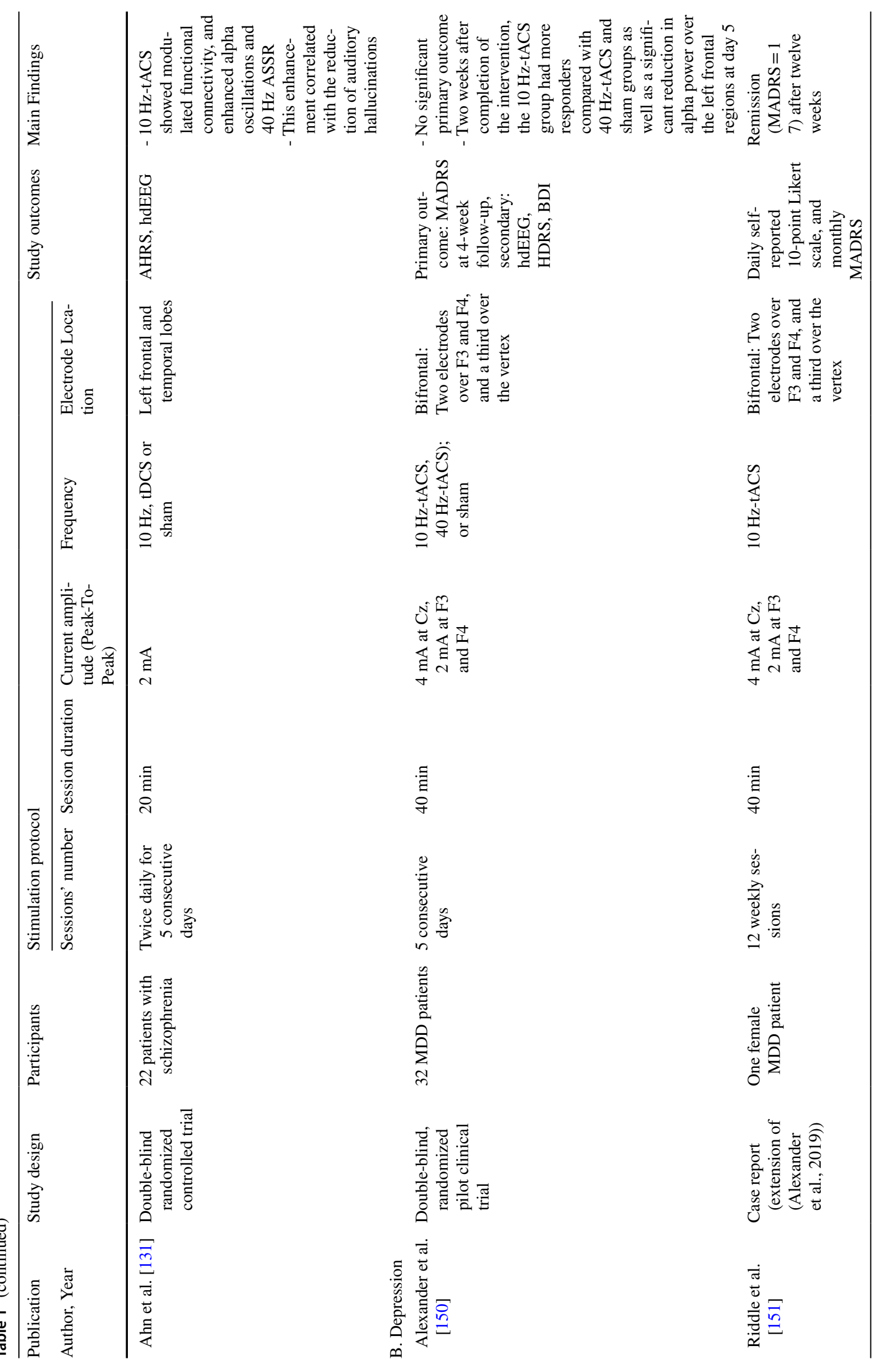




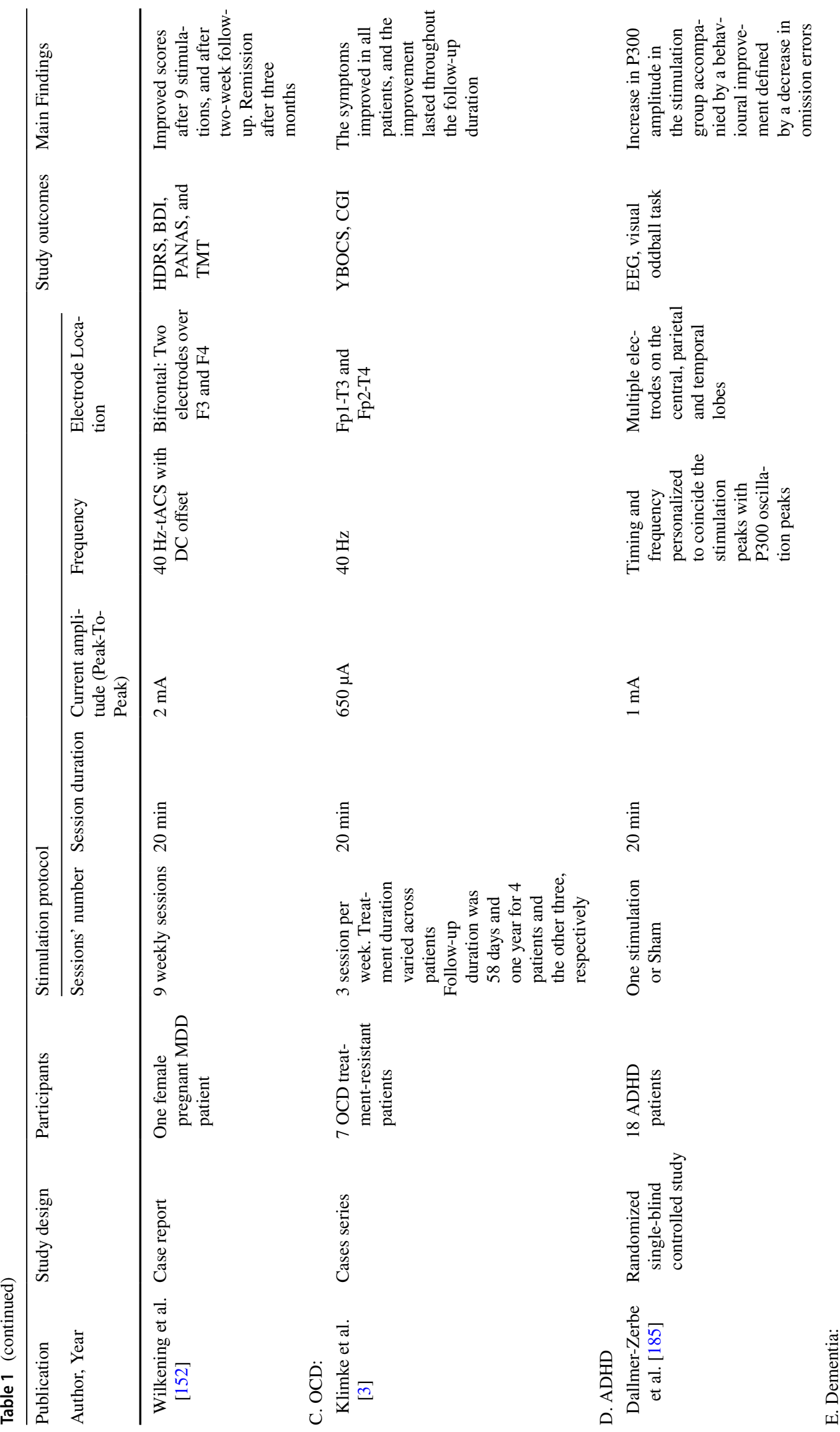




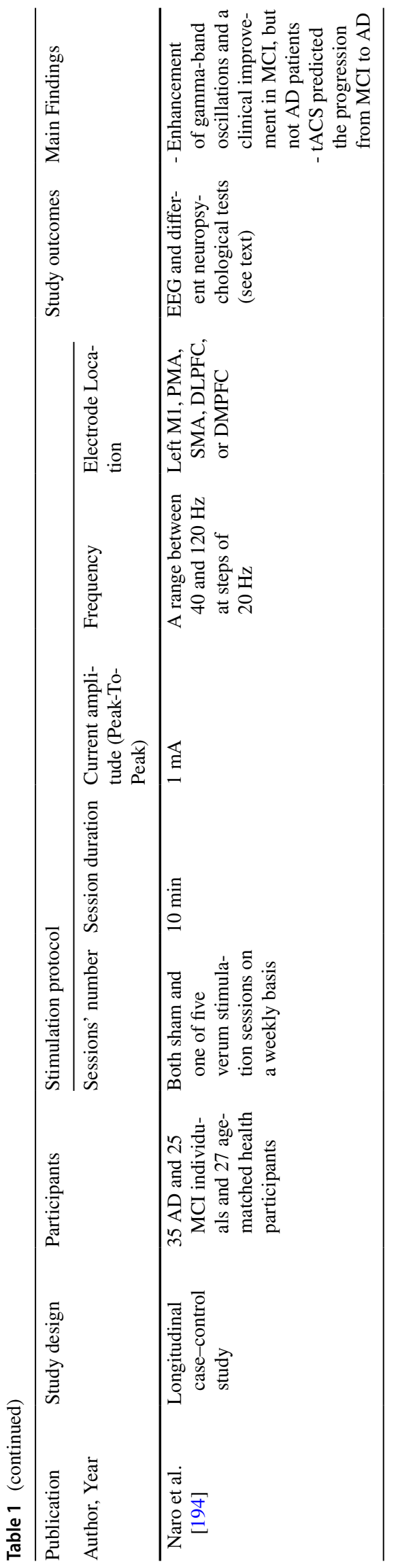

[100]. Most of the studies showing reduced task-related gamma oscillations examined impaired cognitive function [101]. However, there are also reports linking increased stimulus-related gamma-synchrony to positive schizophrenia symptoms [102]. As well, in terms of gamma power, the evoked visual gamma-band response is correlated with positive and disorganised schizophrenia symptoms [103], and even with positive schizotypal personality traits [104]. Moreover, spontaneous gamma oscillations and/or gammaband connectivity measured in resting state conditions are enhanced in patients with schizophrenia [105-113], especially in patients with positive or reality distortion symptoms (hallucinations and delusions) [114].

Revisiting the binding theory of mental representations [115], which assigns mental imagery to the interaction between distant brain regions (a synchronization in the gamma range) [116], such an increase or decrease in gamma oscillations may explain different phenotypes of schizophrenia. For example, the increase in gamma-band power and phase locking (i.e., connectivity) could be related to the emergence of new perceptual representations that are normally absent in healthy individuals, such as auditory hallucinations. In contrast, the decrease in gamma oscillations could be a sign of brain disintegration, and thus, the cause of the impairment or deterioration of normal cognitive functions in normal people. According to these observations, we consider schizophrenia a combination of different clinical presentations that are associated with dysconnectivity [117], not disconnectivity, of the brain, where the differential contributions of different dysconnectional (connection or disconnection) patterns of the patients' brain determine their explicit clinical picture. This functional dysconnectivity is also confirmed by the aberrant underlying anatomical and cellular dysconnectivity [117-119].

In accordance with this assumption, cognitive impairments and negative symptoms in schizophrenia have been linked to reductions in gamma power and phase coherence, whereas positive symptoms are associated with increased gamma activity. In this regard, tACS may help with the diagnosis of and differentiation between different schizophrenic clinical syndromes, in addition to its role in treatment. Future research could exploit tACS-directed characterization of specific oscillatory endotypes in distinct schizophrenic presentations.

One of the main positive symptoms of Schizophrenia is the presence of auditory hallucinations that are sometimes treatment-resistant [120]. In EEG studies, auditory hallucinations in people with schizophrenia are significantly correlated with functional connectivity between both primary auditory cortices. Such enhanced functional connectivity is manifested in the phase synchronization between brain oscillations in both auditory cortices in the range of gamma-band frequencies $[102,121]$. Interestingly, this phenomenon was 
further confirmed by structural changes using diffusion tensor imaging (DTI) [122]. These findings, in turn, support the interhemispheric miscommunication hypothesis of auditory hallucinations [123]. In the light of these discoveries, tACS was able to manipulate auditory perception in healthy participants by decoupling this interhemispheric connectivity. A remarkable finding was that the individual indigenous brain oscillations prior to stimulation dictated the resulting effects of tACS, favouring the use of individually tailored stimulation paradigms [124].

\section{Clinical tACS studies}

Seven publications studied the application of tACS in patients with schizophrenia. They ranged widely in terms of the evidence-based medicine hierarchy. Two case reports and a case series were reported, while the other four publications were clinical trials. Two of them were an openlabel non-controlled trial and a single-blind randomized controlled trial. Finally, two publications comprised a wellstructured double-blind randomized controlled study.

The two case reports were published by the same research group to examine the feasibility of tACS in schizophrenia. They were based on previous findings of reduced theta and gamma oscillation in relation to working memory, especially on the frontal region. One of them showed that one session of 20 min tACS applied to the left DLPFC (F3) and the left posterior parietal region (P3) in theta frequency $(6 \mathrm{~Hz})$, but not gamma $(40 \mathrm{~Hz})$, was able to improve performance in WM task [125]. The same tACS protocol was replicated in the other case report but for five consecutive days, resulting in improved working memory after $6 \mathrm{~Hz}-\mathrm{tACS}$, as well as an improvement in other cognitive domains. After 50 days of follow-up, these effects remained observable [126].

Similarly, the open case series in schizophrenia investigated the efficacy and safety of theta tACS, as theta waves are reduced in the frontal region. The study applied 20 daily tACS sessions for 4 weeks on working days in three subjects with clozapine-resistant schizophrenia. $4.5 \mathrm{~Hz}$-tACS targeted both right and left DLPFCs with an amplitude of $2 \mathrm{~mA}$ for $20 \mathrm{~min}$ per session. Patients were assessed according to their psychiatric clinical symptoms (positive, negative and anxiety), and illness insight, as well as tACS adverse events. They showed a reduction in negative symptoms, anxiety and general psychopathology symptoms with improvement in illness insight [127].

Although the abovementioned case reports obtained results from only a few patients, they may support the feasibility of tACS in schizophrenia research. These effects might be long-term and frequency specific. Furthermore, the case series succeeded in reducing the schizophrenia symptoms and improving insight into treatmentresistant patients without serious side effects. However, well-structured controlled clinical trials are necessary to confirm these findings and to control for the placebo effect. Further research may need to verify modulation of the targeted oscillations using pre- and post-stimulation EEG recordings.

Motivated by the replicated finding of decreased frontal alpha activity in schizophrenia, the open-label non-controlled trial attempted to assess the safety and efficacy of alpha tACS on persistent delusions [128]. The trial recruited 12 patients with schizophrenia who exhibited persistent delusions, despite pharmacological treatment. All patients received two 20-min sessions per day separated by $3 \mathrm{~h}$ for 5 days. Nine of them received stimulations for five more days, and only one participant continued for four more days. tACS of $10 \mathrm{~Hz}$ was applied with $2 \mathrm{~mA}$ intensity via two electrodes over $\mathrm{AFz}$ and $\mathrm{Cz}$. The study aimed to decrease persistent delusions by normalizing alpha oscillations in the medial prefrontal region. The patients were monitored by the Psychiatry Rating Scale (PSYRATS)-Delusions, the Scale for Assessment of Positive Symptoms (SAPS) and the Scale for Assessment of Negative Symptoms (SANS) and were followed up for one month. The results showed a reduction in the delusions, as well as positive and negative symptoms, which was maintained for one month. Interestingly, the patients tolerated the twice-daily regiment without serious side effects.

This study supports the feasibility of twice-daily stimulation and opens the door for the replication of frontal alpha tACS effects on persistent delusions as an add-on option. However, major limitations still exist that should be controlled for in further research. The sample size was small without a placebo control group, and patients were taking different medications, which might have affected the results.

One included publication was a single-blinded, randomized, controlled trial, which attempted to target frontal gamma oscillations that are reduced in schizophrenia. Ten patients with schizophrenia participated in three separate 20-min sessions at least three days apart. They randomly received one of three stimulation protocols: $40 \mathrm{~Hz}$ tACS, tDCS and sham stimulation. $40 \mathrm{~Hz}$ tACS was delivered on the left DLPFC with an amplitude of $2 \mathrm{~mA}$. Meanwhile, they performed a working memory task before, during and after every stimulation session. The study did not report any significant effects of tACS on the task parameters or any serious side effects [129].

Given the small sample size, this study did not exclude the potential rule of gamma tACS on working memory in subjects with schizophrenia. More interestingly, the trial obtained neither pre- nor post-EEG recordings and did not utilize field modelling tools. The lack of an observed effect in response to tACS may be attributed to the difficulties of gamma stimulation per se or to different brain dynamics in patients, especially since the same group managed to 
improve working memory in healthy controls using the same stimulation protocol [130].

The remaining two publications on schizophrenia addressed the first well-structured randomized, double-blind, controlled clinical trial in patients with psychiatric disorders [4, 131]. It investigated the role of alpha tACS based on abnormalities in alpha oscillations over the frontal and temporal regions in schizophrenia. Twenty-two hallucinating participants with schizophrenia were randomized into three groups. While one control group received sham stimulation, the other active ones received either $10 \mathrm{~Hz}$ tACS $(2 \mathrm{~mA})$ or tDCS. Both were applied to the left frontal and temporal lobes. All groups received two 20 min sessions per day for five consecutive days. The primary outcomes were the improvement of auditory hallucinations calculated by the Auditory Hallucination Rating Scale (AHRS) and HighDensity Electroencephalogram (hdEEG). Meanwhile, the secondary outcomes included the Positive and Negative Syndrome Scale (PANSS) and the Brief Assessment of Cognition in Schizophrenia (BACS). Only the group that received $10 \mathrm{~Hz}$ tACS exhibited modulation of functional connectivity, and enhancement of alpha oscillations and the $40 \mathrm{~Hz}$ auditory steady-state response (ASSR). Such enhancement correlated with a reduction in auditory hallucinations as measured by AHRS [131]. However, the primary and secondary clinical outcomes did not reveal significant effects.

This trial is not only the first clinical trial conducted in patients with schizophrenia, but it revealed the ability of tACS to modulate disturbed alpha oscillations in schizophrenia as well $[72,74]$. Interestingly, such a modulation correlated with a reduction in auditory hallucinations as a clinical parameter. Despite this correlation, the clinical outcome did not reach significance. This might be attributed to the small sample size and/or the significant inter-group variation in age, especially since tACS showed the largest effect size for AHRS. Hence, this study requires further replications with a larger sample size and longer duration of follow-up to verify the efficacy of normalization of disturbed alpha oscillations in improving schizophrenia symptoms. Having reported no serious side effects, this study may justify the twice-daily stimulation protocol.

\section{Depression}

Depression, similar to schizophrenia, shows a comparably complex picture of altered brain oscillations [132]. The electrophysiological features of depression manifest some heterogeneity, as well as a greater dependence on the frequency $[132,133]$. Either in terms of the power or the coherence, the low-frequency bands (delta, beta and alpha) were enhanced during the resting-state in depression, in particular, alpha oscillations, which persist even after the transition from closed-eyes to open-eyes states [85, 133-136]. Additionally, the same waves indicated specific connectivity, interhemispheric asymmetries, and even probable prognostic patterns.

In the alpha-band synchrony, the dorsolateral prefrontal cortex (DLPFC) is more connected to the anterior cingulate gyrus [137] and temporal and parietal occipital regions [136]. Alpha-band interhemispheric comparisons revealed frontal alpha asymmetry (FAA) and parietotemporal alpha asymmetry, where the left hemisphere shows more alpha power and local synchrony than the right hemisphere [132, $138,139]$. Interestingly, enhanced alpha activity in depression was associated with better response to antidepressant therapies [140,141]. Though theta waves were also mostly increased in depression, especially within the frontal shortrange functional connections [142], in contrast to alpha waves, the enhanced frontal theta waves were correlated with decreased response [143].

Gamma oscillations differ significantly depending on the state, as they were reduced in the anterior cingulate cortex and in the frontal regions during the resting state and emotional tasks, respectively [144, 145]. Nevertheless, they were enhanced in the frontal and temporal lobes in response to spatial and arithmetic tasks [146]. Interestingly, gamma activity differentiated unipolar depression from bipolar depression according to its power in two different tasks: an auditory task augmented gamma ASSR power in unipolar depression, while an emotional task enhanced temporal and suppressed frontal gamma powers in unipolar depression with respect to bipolar depression [147-149]. Different antidepressant options exhibited either increased or decreased gamma oscillations: serotonergic medications, cognitive therapy and deep brain stimulations dampened them; and in contrast, noradrenergic drugs, ketamine and TMS induced them [145].

\section{Clinical tACS studies}

Three eligible articles examined the application of tACS in depression: one of them is a well-structured double-blind randomized controlled trial, while the other two present two case reports. In the double-blind randomized clinical trial, tACS targeted pathologically increased alpha waves on the left frontal region compared to the right frontal region (i.e., FAA). The study aimed to restore the frontal alpha oscillations by synchronously stimulating both frontal regions. Therefore, 32 patients with Major Depressive Disorder (MDD) were randomly recruited to three study groups: two groups were given two verum tACS protocols $(10 \mathrm{~Hz}-$ tACS or $40 \mathrm{~Hz}-\mathrm{tACS}$ ) with an amplitude of $4 \mathrm{~mA}$ at $\mathrm{Cz}$ and $2 \mathrm{~mA}$ at $\mathrm{F} 3$ and $\mathrm{F} 4$, and the third group received active sham stimulation. The session took $40 \mathrm{~min}$ and was repeated for five consecutive days. The left DLPFC was the stimulation target area, aiming to improve clinical symptoms by retaining its normal alpha frequencies. The primary and secondary 
outcomes included the clinical symptoms applying Montgomery-Asberg Depression Rating Scale (MADRS) at 4-week follow-up and the normalization of alpha oscillations using hdEEG, respectively. Hamilton Depression Rating Scale (HDRS) and the Beck Depression Inventory (BDI) were chosen as exploratory outcomes. The study found no significant results regarding the primary outcome. In terms of MADRS and Hamilton Depression Rating Scale (HDRS), there were more responders at the two-week follow-up in the group that received $10 \mathrm{~Hz}$-tACS. Concerning hdEEG, the same group showed significantly decreased alpha power in the left DLPFC on day 5. Moreover, there were no serious adverse events, manic shift or suicidal ideation induction [150].

This is the first well-structured clinical trial to record an effect of tACS stimulation in some patients with depression who were treated for two weeks by resetting the oscillatory brain disturbances. Such an effect was confined to alpha stimulation but not $40 \mathrm{~Hz}$ stimulation. This supports the idea that only stimulation at a specific frequency alters the oscillatory pattern and thus the behavioural outcome. However, this effect was not maintained after the 4-week follow-up and did not involve the primary outcome. Therefore, a larger number of stimulation sessions and longer follow-up periods should be encouraged since no serious adverse events were detected. Although no FAA was detected in the study sample at baseline, five sessions of $10 \mathrm{~Hz}-\mathrm{t}$ ACS managed to decrease the alpha power over the left frontal region. This might contradict that alpha tACS is supposed to enhance the alpha power as an immediate after-effect. Therefore, further research is needed to identify the possible mechanisms through which alpha tACS decreased the alpha power as a long-term effect. Given the small number of participants in this study, further research should be conducted with a larger sample size.

One of the included case reports on depression was an extension of the previous clinical trial where one participant received extra 12 weekly sessions of $10-\mathrm{Hz}$ tACS. After the original study, the patient showed a response to the treatment without remission. After the 12 sessions, remission was achieved and maintained for at least a 2-month followup [151]. Despite being performed on a single participant, this study might support the feasibility of long-term tACS as a potentially safe tool in MDD treatment research.

The other case report examined the effect of frontal tACS at the gamma frequency band, which is reduced frontally in depressed patients [152]. A pregnant female MDD patient was recruited into the study at week 6 of pregnancy. She received $40 \mathrm{~Hz}$-tACS of $2 \mathrm{~mA}$ over both DLPFCs (F3 and F4) for 20 min per session. After nine weekly stimulation sessions, she was followed-up at two weeks and three months (week 27 of the pregnancy) after the intervention. Her depression condition was monitored by HDRS, BDI,
Positive and Negative Affect Schedule (PANAS) and Trail Making Test (TMT). The study only reported phosphenes without serious side effects. At the end of the nine stimulation sessions and 2-week follow-up, the patient showed improvement in her symptoms and experienced remission after three months. However, it is noteworthy that the same patient showed remission on tDCS used to treat a previous depressive episode. Therefore, the outcome could be attributed to a placebo effect or the stimulation per se, especially because no EEG recordings were done throughout the study [152]. Nonetheless, this case report may encourage further application of tACS in research on pregnant patients as a relatively safer option compared to pharmacotherapeutic agents.

\section{Obsessive-compulsive disorder}

An enormous body of evidence has shown decreased alpha activity in resting state, task-based and symptom-provocation studies in obsessive-compulsive disorder (OCD), especially in frontal areas (hyper-frontality), reasoning the mental overactivity in OCD [139, 153-158]. Interestingly, the location of the resting alpha reduction was subtype-specific: within the frontotemporal areas in doubting OCD subtype; while over the parietooccipital regions in the checking subtype [159]. Unlike depression, alpha asymmetry is not a stable finding in OCD. Nevertheless, it is more pronounced in the doubting OCD with decreased left alpha waves [139, 159]. The decreased alpha activity during the task state may reflect the augmented readiness and/or the preoccupation of OCD patients by obsessions [160]. Surprisingly, alpha synchronization in working memory was reinforced, proposing a compensatory mechanism to inhibit irrelevant information $[139,161]$.

Theta and delta power, especially in frontal regions [157, 162-165], are augmented in OCD [154, 157, 159, 166, 167], where theta augmentation, similar to in depression, is correlated with poor response to treatment $[168,169]$. The findings of beta oscillations were broadly inconsistent [169], but it is thought to be frontally elevated, originating from the anterior cingulate gyrus [166, 167]. Moreover, the frontal beta showed interhemispheric asymmetry with increased activity on the left side [166]. Although gamma-band oscillations are not well studied in OCD, they are thought to be generally decreased [85]. TACS might be used to normalize the altered oscillations, to potentiate the compensatory changes, and to differentiate subtypes in OCD.

\section{Clinical tACS studies}

Only one publication on OCD was included, which discussed a case series of seven treatment-resistant OCD patients. The authors suggested that "DLPFC activity in OCD might be 
pathologically reduced", and thus gamma tACS might lead to its activation. $40 \mathrm{~Hz}$ tACS was administered at both frontotemporal sites (Fp1-T3 and Fp2-T4) to stimulate DLPFC. Patients received three 20-min sessions per week, while the duration of treatment varied across patients from two to seven weeks. All patients were clinically evaluated via the Yale-Brown Obsessive-compulsive Scale (YBOCS) and Clinical Global Impression Scale (CGI) on day 1 before tACS stimulation, and 28 and 56 days later. Three patients were followed up for 1 year. Symptoms improved in all patients, and the improvement lasted throughout the followup duration [3].

The study successfully reduced clinical symptoms of OCD, opening the door for further tACS research in OCD. However, it lacks EEG recordings to evaluate the effect of gamma stimulation on DLPFC. More importantly, it addressed only seven patients with different ages and medication parameters, and the placebo effect cannot be ruled out. Further double-blind controlled studies are necessary to investigate the reproducibility of such an empirical finding.

\section{Bipolar disorder}

Generally, alpha oscillations are inhibited in bipolar patients in many aspects: both resting and evoked alpha waves, with eyes closed or open, and in euthymic or manic participants [170-172]. In contrast, theta and delta oscillatory activity is enhanced [170, 173-175]. Likewise, an enhancement in the beta power response to different stimuli was recorded, which differentiated bipolar disorder from schizophrenia [171, 176-178]. Nonetheless, the beta synchronization was decreased at rest and in response to an auditory stimulation $[179,180]$. Both manic and euthymic patients showed a decreased gamma coherence as evoked by diverse stimuli [96, 148, 181-183]. In summary, the oscillatory changes in bipolar disorder curb the resting-related alpha waves, as well as beta and gamma synchronization, leading to restrained connectivity between brain regions. These two principal findings may explain the disturbed racing thoughts and the distractibility in patients [184] and could be targeted by tACS for normalization. So far, no clinical study investigating the role of tACS in bipolar disorder has been reported according to the systematic search.

\section{Attention-deficit/hyperactivity disorder (ADHD)}

Only one included article addressed ADHD as a singleblind randomized controlled trial. In ADHD, the target P300 amplitude shows some reduction, which is associated with typical cognitive performance deficits in ADHD. Therefore, the trial investigated the role of tACS in potentiating the target $\mathrm{P} 300$ amplitude as disturbed brain oscillations in the theta and delta range. In addition, it aimed to improve cognitive performance in patients with ADHD by such P300 amplification. Therefore, EEG was used to examine brain activity in 18 patients with ADHD underlying a visual oddball paradigm. During the task, $1 \mathrm{~mA}$ tACS was applied for 20 min through multiple electrodes on the central, parietal and temporal lobes. The stimulation timing and frequency were personalized so that the stimulation peaks could coincide with P300 oscillation peaks to amplify them. The study found P300 amplitude to be significantly augmented in the verum group compared to the sham condition. Interestingly, this P300 amplification was accompanied by a behavioural improvement in task performance. No serious adverse events were reported [185].

This clinical trial is not only the first study to support research on the role of tACS in ADHD, but it also substantiates tACS applicability to modulate event-related potentials for clinical relevance. Nonetheless, future studies could replicate it with more patients and compare tACS effects in patients and healthy controls, especially because a similar stimulation protocol did manage to alter P300 parameters in healthy participants [186]. However, this study paved the way for the feasibility of tACS to modulate event-related potential components with subsequent behavioural improvement without serious side effects.

\section{Dementia}

To the best of our knowledge, there is no available study using tACS as a treatment in either Alzheimer's disease (AD) or dementia. Only a single included study examined the role of tACS as a prognostic factor in predicting the progression of patients with mild cognitive impairment (MCI) to AD. The study was based on findings of decreased gamma-band connectivity in AD [187-192] and increased local gamma-band power in contrast to MCI during resting and task conditions [193]. The study questioned whether the response to gamma tACS could differentiate MCI from $\mathrm{AD}$ and predict the progression of $\mathrm{MCI}$ to $\mathrm{AD}$. The authors recruited $35 \mathrm{AD}$ and $25 \mathrm{MCI}$ individuals, as well as 27 age-matched healthy participants, and followed them up for 2 years. On a weekly basis, each participant randomly received both sham stimulation over the left primary motor area (M1) and five verum stimulation sessions. Gammaband tACS was applied for $10 \mathrm{~min}$ to one of the following sites per verum session: M1, premotor area (PMA), supplementary motor area (SMA), DLPFC, or the dorsomedial prefrontal cortex (DMPFC). Stimulation of $1 \mathrm{~mA}$ was set to vary continuously and randomly in a range between 40 and $120 \mathrm{~Hz}$ at steps of $20 \mathrm{~Hz}$, with zero-degree phase-lag. EEG was recorded before and after each session in addition to a follow-up EEG after 2 years.

tACS caused enhancement of gamma-band oscillations and a clinical improvement according to different 
neuropsychological tests in MCI patients. AD patients, however, showed neither. After 2 years, a group of MCI patients progressed to $\mathrm{AD}$. Interestingly, this group did not report any tACS after-effects before the 2-year follow-up period [194]. The sessions were well tolerated by the patients.

This is the first publication to report the use of tACS on dementia. Its results might help to identify the potential role of tACS in the differential diagnosis of MCI and AD and in prognosis prediction for MCI. Nevertheless, it is still far from providing a biomarker for dementia progression given the discrepancy between connectivity within and local power of gamma oscillations. Further research should replicate this study in larger sample sizes to verify the reproducibility of these findings. Moreover, follow-up time points should be encouraged with EEG recordings to obtain electrophysiological markers of the disease progression.

\section{General discussion}

To the best of our knowledge, this is the first systematic literature search to report studies that apply tACS in clinical psychiatric research. tACS publications on five different psychiatric disorders were reported. First, in schizophrenia, frontal alpha tACS was promising as an add-on treatment for persistent delusions [128] and was capable of normalizing disturbed alpha oscillations correlated with a decrease in auditory hallucinations $[4,131]$. On the other hand, frontal gamma tACS has not shown effects so far [125, 129]. Case reports revealed that frontal theta tACS could be helpful for improving clinical symptoms, even in schizophrenia with clozapine resistance [125-127]. Second, in depression, bifrontal alpha tACS was able to normalize alpha oscillations and concurrently showed a higher response rate for at least two weeks [150]. Two case reports showed that tACS might be safely tolerated, even for a large number of sessions or during pregnancy $[151,152]$. Third, a case series in OCD with seven patients revealed an empirical improvement in symptoms after frontal gamma tACS [3]. Fourth, a singleblind trial managed to amplify P300 in ADHD patients with subsequent improvement in working memory task [185]. Finally, frontal gamma tACS might support a potential role for tACS as a diagnostic or prognostic tool in MCI and AD [194]. All the studies reported neither serious side effects nor exacerbation of clinical symptoms (i.e., manic shift or worsening of the clinical phenotype) $[3,4,125-129,131$, $150-152,185]$

Despite the very limited number of studies applying tACS in psychiatry, its efficiency seems promising in the psychiatric field keeping in mind the following considerations: (1) some studies have recruited treatment-resistant patients, and managed to diminish their symptoms [3, 127]; (2) the relatively good safety profile of tACS may advocate twice-daily stimulations, use in pregnancy, or large stimulation doses without fear of clinical worsening [128, 131, 150-152]; (3) the novelty of its treatment approach targeting specific alterations of brain functions at specific locations makes it significantly differ from conventional treatment strategies, such as pharmacotherapy. Although psychotropic drugs are widely accepted and approved in psychiatry, they still pose substantial problems with resistance, compliance and safety profile [195-197]; (4) tACS could help to target baseline oscillations, as well as event-related potentials [185]; (5) utilization of tACS should not be restricted to therapeutic purposes. The reactivity of the brain circuits to the stimulation protocol could serve as a possible tool to determine the diagnosis, classification or prognosis of psychiatric disorders, for instance, in patients with MCI [194]. However, tACS in psychiatric research is still far from being approved as a reliable tool for the management of psychiatric conditions. Further research is necessary to replicate these findings and to answer important questions.

An intriguing problem might be whether disturbed brain oscillations are causally related to the disorder or represent just an association. Moreover, it is not yet clear whether tACS can be investigated as a stand-alone therapeutic tool or rather an add-on option to target specific residual symptoms. The concomitant use of drugs while applying tACS could complicate future research. Drug-naïve and drugnon-compliant patients could be recruited to rule out effects of the drugs or to prove a synergistic or antagonistic effect between both tACS and pharmacotherapy. Along with this, the therapeutic role of tACS should be precisely defined with respect to whether it can improve the whole clinical picture of psychiatric illness [150] or is confined to targeting a specific modality of symptoms [185] that did not respond to other therapeutic options. Referring to the phenomenon of Arnold tongues, the weak tACS current could entrain the brain activity associated with a certain brain state when the stimulation targets these brain oscillations at their frequency. Hence, a certain brain state may be induced in participants to maximize these oscillations, so they can easily be entrained by tACS (i.e., state-dependent stimulation) [198]. In that sense, tACS could be initially used to target a pattern of brain oscillations driven by a single modality or symptom of psychiatric disorders. This was shown to be possible in targeting P300 amplitude driven by WM in ADHD and gamma oscillations induced by WM in MCI $[185,194]$.

The discrepancy of brain dynamics and response to tACS between healthy participants and patients constitutes an enigma that should be considered during replication of similar stimulation protocols. Healthy participants may be more susceptible to specific tACS stimulation protocols than patients with psychiatric disorders $[129,130]$ or vice versa $[185,186]$. Similarly, acute and chronic patients may show distinct tACS responses, especially tACS after-effects, which 
necessitates long-term plasticity that might be affected in chronic patients [199]. Future research may try to determine potential responders to tACS based on their electrophysiological markers or clinical phenotype.

In light of these future research questions, several points could be highlighted. The more individualized the stimulation protocol is designed, the more effectively it normalizes the disturbed oscillations, for example, in the schizophrenic case reports and the ADHD study [125, 126, 185]. tACS effects do not depend on the direct application of a specific frequency per se but on modulation of the pre-existing endogenous oscillations that are coupled with the specific brain state. Accordingly, it might be helpful to personalize future stimulation paradigms, by individually defining disturbed EEG patterns regarding frequency and localization of interest prior to stimulation and then tailoring the stimulation parameters (frequency and location of stimulation) accordingly [200, 201]. Such personalisation of the stimulation protocols stands in accordance with previous findings of the heritable electrophysiological endophenotypes associated with some psychiatric disorders [202, 203]. For this, tACS modelling techniques might be recommended to optimize stimulation parameters and obtain better results [13]. Overall, in the same context, electrophysiological recording via EEG or MEG could be valuable to observe achievement of the desired change in brain electrical activity and to individualize the stimulation protocol [204]. Finally, multicentre double-blind clinic trials could be fostered to investigate larger sample sizes. This may also facilitate the utility of different stimulation protocols in different patients or within the same individuals to rule out a stimulation effect per se $[4,125,131,150]$ and to question the potential synergism of different stimulation parameters, respectively (for more technical guidance: [12, 205-207]).

Despite the relative safety profile of tACS, several side effects could coincide with the stimulation period: phosphenes, dizziness, headache and skin sensations, such as tingling, itching, etc.[205]. Phosphenes tend to be more frequent upon frontal montages due to retinal stimulation. On the other hand, dizziness is more common in posterior montages due to vestibular stimulation. Skin adverse events, as well as phosphenes, frequently co-occur with higher frequencies and/or intensities [208-210]. Headache could outlast the stimulation phase, especially with longer stimulation duration [211, 212]. In contrast to tDCS, tACS induces less serious (e.g., epilepsy) and less persistent (e.g., burn and dermatitis) adverse events [208, 213].

To display a balanced overview of the current insufficient knowledge on tACS, two major limitations should be presented: (1) some studies failed to show an effect in both healthy participants and patients, and (2) entrainment of brain oscillations is confounded by other proposed mechanisms of action. The individual functional as well as the structural variability of the brain, the wide range of stimulation parameters and technical difficulties could explain why tACS failed to alter the behavioural outcomes [67, 129, 186, 214, 215]. In this regard, strict modelling techniques and peri-stimulation EEG recordings could decipher the inability of tACS to induce electrophysiological or behavioural outcomes. Consistently, this limitation is complicated by the obstacle of stimulation artefact rejection in EEG or MEG [216, 217]. Future research may be needed to identify optimal tACS parameters to ensure a consecutive effect.

A second major drawback of tACS is the indirect mechanisms of action rather than direct entrainment of endogenous brain oscillations. Stimulation of peripheral nerves and the retina could account for the entrainment of brain oscillations [213, 218-222]. Similarly, cranial electrotherapy stimulation, a close alternating current stimulation tool applied on the forehead and mastoids, induces electrical brain changes via direct stimulation of cranial nerves [223, 224]. Although this hypothesis of the retina and peripheral somatosensory stimulation cannot be fully excluded, evidence supports the direct causality of tACS to entrain brain oscillations [20, 222, 225]. Consistent with this conclusion, tACS revealed frequency-, phase- and montage- and state-specific effects $[67,198,222,226]$. Further research could try to estimate the contribution of these indirect mechanisms to the whole tACS using modelling techniques, as well as active control groups [222].

\section{Conclusions}

tACS, a unique form of NIBS, results in both online and offline brain changes by entraining brain oscillations and inducing neuroplasticity, respectively. It has been extensively used to alter electrophysiological brain activity, and thus cognitive functions in healthy participants. Similarly, disturbed brain oscillations in psychiatric conditions may constitute a potential target for modulation by tACS without major adverse events. Its first few applications in psychiatry seem promising and encouraging for more research to discover its full potential with respect to therapeutic and diagnostic roles. Given its safety profile, these first studies may support tACS feasibility in altering disturbed brain oscillations, thus improving behavioural outcomes. However, further well-structured double-blind controlled trials with larger sample sizes and longer follow-up durations are still needed to replicate the current findings. They may help to detect response predictors and control for various confounding factors. In this regard, electrophysiological recordings, as well as modelling techniques, are encouraged to optimize stimulation protocols and to detect possible factors contributing to the effects of tACS. 
Acknowledgments We would like to thank American Journal Experts (AJE) for English language editing.

Author contributions OE: wrote the manuscript, conducted the systematic search, and designed the table and the PRISMA chart. GL: wrote the manuscript, and carefully reviewed it. $\mathrm{CSH}$ : wrote the manuscript, and carefully reviewed it. CM: wrote the manuscript, carefully reviewed it, and supervised the whole work.

Funding Open Access funding enabled and organized by Projekt DEAL. This work was funded by the German Research Foundation (DFG: www.dfg.de) through two projects: (1) the Collaborative Research Centre SFB936-Project C6-Third Funding Period to C. Mulert and G. Leicht, and (2) the Collaborative Research Centre SFB/ TRR135-Project B7-Second Funding Period to C. Mulert.

\section{Compliance with ethical standards}

Conflicts of interest CSH holds a patent on brain stimulation. The other authors declare no competing interests.

Open Access This article is licensed under a Creative Commons Attribution 4.0 International License, which permits use, sharing, adaptation, distribution and reproduction in any medium or format, as long as you give appropriate credit to the original author(s) and the source, provide a link to the Creative Commons licence, and indicate if changes were made. The images or other third party material in this article are included in the article's Creative Commons licence, unless indicated otherwise in a credit line to the material. If material is not included in the article's Creative Commons licence and your intended use is not permitted by statutory regulation or exceeds the permitted use, you will need to obtain permission directly from the copyright holder. To view a copy of this licence, visit http://creativecommons.org/licenses/by/4.0/.

\section{References}

1. Marshall L, Helgadóttir H, Mölle M, Born J (2006) Boosting slow oscillations during sleep potentiates memory. Nature 444:610-613. https://doi.org/10.1038/nature05278

2. Antal A, Boros K, Poreisz C et al (2008) Comparatively weak after-effects of transcranial alternating current stimulation (tACS) on cortical excitability in humans. Brain Stimul 1:97105. https://doi.org/10.1016/j.brs.2007.10.001

3. Klimke A, Nitsche MA, Maurer K, Voss U (2016) Case report: successful treatment of therapy-resistant OCD with application of transcranial alternating current stimulation (tACS). Brain Stimul 9:463-465. https://doi.org/10.1016/j.brs.2016.03.005

4. Mellin JM, Alagapan S, Lustenberger C et al (2018) Randomized trial of transcranial alternating current stimulation for treatment of auditory hallucinations in schizophrenia. Eur Psychiatry 51:25-33. https://doi.org/10.1016/j.eurpsy.2018.01.004

5. Ruffini G, Wendling F, Merlet I et al (2013) Transcranial current brain stimulation (tCS): Models and technologies. IEEE Trans Neural Syst Rehabil Eng 21:333-345. https://doi.org/10.1109/ TNSRE.2012.2200046

6. Antal A, Paulus W (2013) Transcranial alternating current stimulation (tACS). Front Hum Neurosci 7:317. https://doi. org/10.3389/fnhum.2013.00317

7. Herrmann CS, Strüber D, Helfrich RF, Engel AK (2016) EEG oscillations: from correlation to causality. Int J Psychophysiol 103:12-21
8. Bonnefond M, Kastner S, Jensen O (2017) Communication between brain areas based on nested oscillations. eNeuro 2017:4. https://doi.org/10.1523/ENEURO.0153-16.2017

9. Fries P (2005) A mechanism for cognitive dynamics: neuronal communication through neuronal coherence. Trends Cogn Sci 9:474-480. https://doi.org/10.1016/j.tics.2005.08.011

10. Fehér KD, Morishima Y (2016) Concurrent electroencephalography recording during transcranial alternating current stimulation (tACS). J Vis Exp 2016:e53527. https://doi.org/10.3791/53527

11. Schestatsky P, Morales-Quezada L, Fregni F (2013) Simultaneous EEG monitoring during transcranial direct current stimulation. J Vis Exp 2013:e50426. https://doi.org/10.3791/50426

12. Woods AJ, Antal A, Bikson M et al (2016) A technical guide to tDCS, and related non-invasive brain stimulation tools. Clin Neurophysiol 127:1031-1048

13. Huang Y, Datta A, Bikson M, Parra LC (2018) ROAST: An open-source, fully-automated, realistic volumetric-approachbased simulator for TES. In: Proceedings of the annual international conference of the IEEE engineering in medicine and biology society, EMBS. Institute of Electrical and Electronics Engineers Inc, pp 3072-3075

14. Vosskuhl J, Strüber D, Herrmann CS (2018) Non-invasive brain stimulation: a paradigm shift in understanding brain oscillations. Front Hum Neurosci 12:1-19. https://doi.org/10.3389/fnhum 2018.00211

15. Alekseichuk I, Falchier AY, Linn G et al (2019) Electric field dynamics in the brain during multi-electrode transcranial electric stimulation. Nat Commun 10:1-10. https://doi.org/10.1038/ s41467-019-10581-7

16. Vöröslakos M, Takeuchi Y, Brinyiczki K et al (2018) Direct effects of transcranial electric stimulation on brain circuits in rats and humans. Nat Commun 9:1-17. https://doi.org/10.1038/ s41467-018-02928-3

17. Schwab BC, Misselhorn J, Engel AK (2019) Modulation of largescale cortical coupling by transcranial alternating current stimulation. Brain Stimul 12:1187-1196. https://doi.org/10.1016/j. brs.2019.04.013

18. Weinrich CA, Brittain JS, Nowak M et al (2017) Modulation of long-range connectivity patterns via frequency-specific stimulation of human cortex. Curr Biol 27:3061-3068.e3. https://doi. org/10.1016/j.cub.2017.08.075

19. Antal A, Herrmann CS (2016) Transcranial alternating current and random noise stimulation: possible mechanisms. Neural Plast. https://doi.org/10.1155/2016/3616807

20. Krause MR, Vieira PG, Csorba BA et al (2019) Transcranial alternating current stimulation entrains single-neuron activity in the primate brain. Proc Natl Acad Sci USA 116:5747-5755. https ://doi.org/10.1073/pnas.1815958116

21. Reato D, Rahman A, Bikson M, Parra LC (2010) Low-intensity electrical stimulation affects network dynamics by modulating population rate and spike timing. J Neurosci 30:15067-15079. https://doi.org/10.1523/JNEUROSCI.2059-10.2010

22. Radman T, Ramos RL, Brumberg JC, Bikson M (2009) Role of cortical cell type and morphology in subthreshold and suprathreshold uniform electric field stimulation in vitro. Brain Stimul 2:215. https://doi.org/10.1016/j.brs.2009.03.007

23. Fröhlich F (2014) Endogenous and exogenous electric fields as modifiers of brain activity: rational design of noninvasive brain stimulation with transcranial alternating current stimulation. Dialog Clin Neurosci 16:93-102

24. Ulrich D (2002) Dendritic resonance in rat neocortical pyramidal cells. J Neurophysiol 87:2753-2759. https://doi.org/10.1152/ jn.2002.87.6.2753

25. Spruston N (2008) Pyramidal neurons: dendritic structure and synaptic integration. Nat Rev Neurosci 9:206-221 
26. Kim EJ, Juavinett AL, Kyubwa EM et al (2015) Three types of cortical layer 5 neurons that differ in brain-wide connectivity and function. Neuron 88:1253-1267. https://doi.org/10.1016/j.neuro n.2015.11.002

27. Koganemaru S, Mikami Y, Matsuhashi M et al (2019) Cerebellar transcranial alternating current stimulation modulates human gait rhythm. Neurosci Res. https://doi.org/10.1016/j.neure s.2019.12.003

28. Liu A, Vöröslakos M, Kronberg G et al (2018) Immediate neurophysiological effects of transcranial electrical stimulation. Nat Commun. https://doi.org/10.1038/s41467-018-07233-7

29. Deans JK, Powell AD, Jefferys JGR (2007) Sensitivity of coherent oscillations in rat hippocampus to AC electric fields. J Physiol 583:555-565. https://doi.org/10.1113/jphysiol.2007.137711

30. Ali MM, Sellers KK, Fröhlich F (2013) Transcranial alternating current stimulation modulates large-scale cortical network activity by network resonance. J Neurosci 33:11262-11275. https://doi.org/10.1523/JNEUROSCI.5867-12.2013

31. Schmidt SL, Iyengar AK, Foulser AA et al (2014) Endogenous cortical oscillations constrain neuromodulation by weak electric fields. Brain Stimul 7:878-889. https://doi.org/10.1016/j. brs.2014.07.033

32. Markram H, Lübke J, Frotscher M, Sakmann B (1997) Regulation of synaptic efficacy by coincidence of postsynaptic APs and EPSPs. Science (80) 275:213-215. https://doi.org/10.1126/ science.275.5297.213

33. Zaehle T, Rach S, Herrmann CS (2010) Transcranial alternating current stimulation enhances individual alpha activity in human EEG. PLoS ONE 5:e13766. https://doi.org/10.1371/ journal.pone.0013766

34. Vossen A, Gross J, Thut G (2015) Alpha power increase after transcranial alternating current stimulation at alpha frequency (a-tACS) reflects plastic changes rather than entrainment. Brain Stimul 8:499-508. https://doi.org/10.1016/j.brs.2014.12.004

35. Guerra A, Suppa A, Bologna M et al (2018) Boosting the LTP-like plasticity effect of intermittent theta-burst stimulation using gamma transcranial alternating current stimulation. Brain Stimul 11:734-742. https://doi.org/10.1016/j. brs.2018.03.015

36. Wischnewski M, Engelhardt M, Salehinejad MA et al (2019) NMDA receptor-mediated motor cortex plasticity after 20 $\mathrm{Hz}$ transcranial alternating current stimulation. Cereb Cortex 29:2924-2931. https://doi.org/10.1093/cercor/bhy 160

37. Kasten FH, Dowsett J, Herrmann CS (2016) Sustained aftereffect of $\alpha$-tACS lasts up to 70 min after stimulation. Front Hum Neurosci. https://doi.org/10.3389/fnhum.2016.00245

38. Nitsche MA, Paulus W (2000) Excitability changes induced in the human motor cortex by weak transcranial direct current stimulation. J Physiol 527:633-639. https://doi. org/10.1111/j.1469-7793.2000.t01-1-00633.x

39. Kabakov AY, Muller PA, Pascual-Leone A et al (2012) Contribution of axonal orientation to pathway-dependent modulation of excitatory transmission by direct current stimulation in isolated rat hippocampus. J Neurophysiol 107:1881-1889. https://doi. org/10.1152/jn.00715.2011

40. Rahman A, Reato D, Arlotti M et al (2013) Cellular effects of acute direct current stimulation: somatic and synaptic terminal effects. J Physiol 591:2563-2578. https://doi.org/10.1113/jphys iol.2012.247171

41. Song M, Shin Y, Yun K (2014) Beta-frequency EEG activity increased during transcranial direct current stimulation. NeuroReport 25:1433-1436. https://doi.org/10.1097/WNR.00000 00000000283

42. Nitsche MA, Fricke K, Henschke U et al (2003) Pharmacological modulation of cortical excitability shifts induced by transcranial direct current stimulation in humans. J Physiol 553:293-301. https://doi.org/10.1113/jphysiol.2003.049916

43. Nitsche MA, Grundey J, Liebetanz D, Lang N, Frithjof Tergau WP (2004) Catecholaminergic consolidation of motor cortical neuroplasticity in humans. Cereb Cortex 14:1240-1245. https:// doi.org/10.1093/cercor/bhh085

44. Bikson M, Inoue M, Akiyama $\mathrm{H}$ et al (2004) Effect of uniform extracellular DC electric fields on excitability in rat hippocampal slices in vitro. J Physiol 557:175-190. https://doi.org/10.1113/ jphysiol.2003.055772

45. Ardolino G, Bossi B, Barbieri S, Priori A (2005) Non-synaptic mechanisms underlie the after-effects of cathodal transcutaneous direct current stimulation of the human brain. J Physiol 568:653663. https://doi.org/10.1113/jphysiol.2005.088310

46. Kanai R, Chaieb L, Antal A et al (2008) Frequency-dependent electrical stimulation of the visual cortex. Curr Biol 18:1839 1843. https://doi.org/10.1016/j.cub.2008.10.027

47. Jackson MP, Rahman A, Lafon B et al (2016) Animal models of transcranial direct current stimulation: methods and mechanisms. Clin Neurophysiol 127:3425-3454. https://doi. org/10.1016/j.clinph.2016.08.016

48. Stagg CJ, Antal A, Nitsche MA (2018) Physiology of transcranial direct current stimulation. J ECT 34:144-152. https://doi. org/10.1097/YCT.0000000000000510

49. Lefaucheur JP, Wendling F (2019) Mechanisms of action of tDCS: a brief and practical overview. Neurophysiol Clin 49:269-275. https://doi.org/10.1016/j.neucli.2019.07.013

50. Chase HW, Boudewyn MA, Carter CS, Phillips ML (2020) Transcranial direct current stimulation: a roadmap for research, from mechanism of action to clinical implementation. Mol Psychiatry 25:397-407. https://doi.org/10.1038/s4138 0-019-0499-9

51. Yavari F, Jamil A, Mosayebi Samani M et al (2018) Basic and functional effects of transcranial electrical stimulation (tES) an introduction. Neurosci Biobehav Rev 85:81-92. https://doi. org/10.1016/j.neubiorev.2017.06.015

52. Horvath JC, Mathews J, Demitrack MA, Pascual-Leone A (2010) The neurostar TMS device: conducting the FDA approved protocol for treatment of depression. J Vis Exp. https ://doi.org/10.3791/2345

53. FDA permits marketing of transcranial magnetic stimulation for treatment of obsessive compulsive disorder|FDA (2020) https://www.fda.gov/news-events/press-announcements/fdapermits-marketing-transcranial-magnetic-stimulation-treat ment-obsessive-compulsive-disorder. Accessed 12 Jun 2020

54. McClintock SM, Reti IM, Carpenter LL et al (2018) Consensus recommendations for the clinical application of repetitive transcranial magnetic stimulation (rTMS) in the treatment of depression. J Clin Psychiatry 79:35-48

55. Barker AT, Jalinous R, Freeston IL (1985) Non-invasive magnetic stimulation of human motor cortex. Lancet 325:1106-1107

56. Barker AT, Shields K (2017) Transcranial magnetic stimulation: basic principles and clinical applications in migraine. Headache 57:517-524. https://doi.org/10.1111/head.13002

57. Burke D, Hicks R, Gandevia SC et al (1993) Direct comparison of corticospinal volleys in human subjects to transcranial magnetic and electrical stimulation. J Physiol 470:383-393. https:// doi.org/10.1113/jphysiol.1993.sp019864

58. Cirillo G, Di Pino G, Capone F et al (2017) Neurobiological after-effects of non-invasive brain stimulation. Brain Stimul 10:1-18. https://doi.org/10.1016/j.brs.2016.11.009

59. Rothwell J, Thompson P, Day B et al (1991) Stimulation of the human motor cortex through the scalp. Exp Physiol 76:159-200. https://doi.org/10.1113/expphysiol.1991.sp003485 
60. Terao Y, Ugawa Y (2002) Basic mechanisms of TMS. J Clin Neurophysiol 19:322-343

61. Hallett M (2007) Transcranial magnetic stimulation: a primer. Neuron 55:187-199. https://doi.org/10.1016/j.neuro n.2007.06.026

62. Funke K, Benali A (2011) Modulation of cortical inhibition by rTMS-findings obtained from animal models. J Physiol 589:4423-4435. https://doi.org/10.1113/jphysiol.2011.206573

63. Klomjai W, Katz R, Lackmy-Vallée A (2015) Basic principles of transcranial magnetic stimulation (TMS) and repetitive TMS (rTMS). Ann Phys Rehabil Med 58:208-213. https://doi. org/10.1016/j.rehab.2015.05.005

64. Bland NS, Sale MV (2019) Current challenges: the ups and downs of tACS. Exp Brain Res 237:3071-3088. https://doi. org/10.1007/s00221-019-05666-0

65. Tavakoli AV, Yun K (2017) Transcranial alternating current stimulation (tACS) mechanisms and protocols. Front Cell Neurosci 11:214

66. Fröhlich F, Sellers KK, Cordle AL (2014) Targeting the neurophysiology of cognitive systems with transcranial alternating current stimulation. Expert Rev Neurother 15:145-167

67. Schutter DJLG, Wischnewski M (2016) A meta-analytic study of exogenous oscillatory electric potentials in neuroenhancement. Neuropsychologia 86:110-118. https://doi.org/10.1016/j.neuro psychologia.2016.04.011

68. Moher D, Liberati A, Tetzlaff J et al (2009) Preferred reporting items for systematic reviews and meta-analyses: the PRISMA statement. PLoS Med 2009:6

69. Uhlhaas PJ, Singer W (2010) Abnormal neural oscillations and synchrony in schizophrenia. Nat Rev Neurosci 11:100-113

70. Uhlhaas PJ, Haenschel C, Nikolić D, Singer W (2008) The role of oscillations and synchrony in cortical networks and their putative relevance for the pathophysiology of schizophrenia. Schizophr Bull 34:927-943. https://doi.org/10.1093/schbul/sbn062

71. Ahmed AO, Strauss GP, Buchanan RW et al (2018) Schizophrenia heterogeneity revisited: clinical, cognitive, and psychosocial correlates of statistically-derived negative symptoms subgroups. J Psychiatr Res 97:8-15. https://doi.org/10.1016/j.jpsychires .2017.11.004

72. Hinkley LBN, Vinogradov S, Guggisberg AG et al (2011) Clinical symptoms and alpha band resting-state functional connectivity imaging in patients with schizophrenia: Implications for novel approaches to treatment. Biol Psychiatry 70:1134-1142. https:// doi.org/10.1016/j.biopsych.2011.06.029

73. Omori M, Koshino Y, Murata T et al (1995) Quantitative EEG in never-treated schizophrenic patients. Biol Psychiatry 38:303309. https://doi.org/10.1016/0006-3223(95)00300-6

74. Sponheim SR, Clementz BA, Iacono WG, Beiser M (2000) Clinical and biological concomitants of resting state EEG power abnormalities in schizophrenia. Biol Psychiatry 48:1088-1097. https://doi.org/10.1016/s0006-3223(00)00907-0

75. Goldstein MR, Peterson MJ, Sanguinetti JL et al (2015) Topographic deficits in alpha-range resting EEG activity and steady state visual evoked responses in schizophrenia. Schizophr Res 168:145-152. https://doi.org/10.1016/j.schres.2015.06.012

76. Jia S, Liu M, Huang P et al (2019) Abnormal alpha rhythm during self-referential processing in schizophrenia patients. Front Psychiatry 10:691. https://doi.org/10.3389/fpsyt.2019.00691

77. Siekmeier PJ, Stufflebeam SM (2010) Patterns of spontaneous magnetoencephalographic activity in patients with schizophrenia. J Clin Neurophysiol 27:179-190. https://doi.org/10.1097/ WNP.0b013e3181e0b20a

78. Boutros NN, Arfken C, Galderisi S et al (2008) The status of spectral EEG abnormality as a diagnostic test for schizophrenia. Schizophr Res 99:225-237
79. Moran LV, Hong LE (2011) High vs low frequency neural oscillations in schizophrenia. Schizophr Bull 37:659-663. https://doi. org/10.1093/schbul/sbr056

80. Tauscher J, Fischer P, Neumeister A et al (1998) Low frontal electroencephalographic coherence in neuroleptic-free schizophrenic patients. Biol Psychiatry 44:438-447. https://doi. org/10.1016/S0006-3223(97)00428-9

81. Schmiedt C, Brand A, Hildebrandt H, Basar-Eroglu C (2005) Event-related theta oscillations during working memory tasks in patients with schizophrenia and healthy controls. Cogn Brain Res 25:936-947. https://doi.org/10.1016/j.cogbrainres.2005.09.015

82. Haenschel C, Bittner RA, Waltz J et al (2009) Cortical oscillatory activity is critical for working memory as revealed by deficits in early-onset schizophrenia. J Neurosci 29:9481-9489. https://doi. org/10.1523/JNEUROSCI.1428-09.2009

83. Shreekantiah Umesh D, Tikka SK, Goyal N et al (2016) Resting state theta band source distribution and functional connectivity in remitted schizophrenia. Neurosci Lett 630:199-202. https:// doi.org/10.1016/j.neulet.2016.07.055

84. Andreou C, Leicht G, Nolte G et al (2015) Resting-state thetaband connectivity and verbal memory in schizophrenia and in the high-risk state. Schizophr Res 161:299-307. https://doi. org/10.1016/j.schres.2014.12.018

85. Newson JJ, Thiagarajan TC (2019) EEG Frequency bands in psychiatric disorders: a review of resting state studies. Front Hum Neurosci 12:1-24. https://doi.org/10.3389/fnhum .2018 .00521

86. Uhlhaas PJ, Linden DEJ, Singer W et al (2006) Dysfunctional long-range coordination of neural activity during gestalt perception in schizophrenia. J Neurosci 26:8168-8175. https://doi. org/10.1523/JNEUROSCI.2002-06.2006

87. Uhlhaas PJ, Singer W (2013) High-frequency oscillations and the neurobiology of schizophrenia. Dialogues Clin Neurosci $15: 301-313$

88. Lisman J (2012) Excitation, inhibition, local oscillations, or large-scale loops: What causes the symptoms of schizophrenia? Curr Opin Neurobiol 22:537-544

89. Hirvonen J, Wibral M, Palva JM et al (2017) Whole-brain sourcereconstructed MEG-data reveal reduced long-range synchronization in chronic schizophrenia. eNeuro 2017:4. https://doi. org/10.1523/ENEURO.0338-17.2017

90. Green MF, Mintz J, Salveson D et al (2003) Visual masking as a probe for abnormal gamma range activity in schizophrenia. Biol Psychiatry 53:1113-1119. https://doi.org/10.1016/S0006 -3223(02)01813-9

91. Kwon JS, O’Donnell BF, Wallenstein GV et al (1999) Gamma frequency-range abnormalities to auditory stimulation in schizophrenia. Arch Gen Psychiatry 56:1001-1005. https://doi. org/10.1001/archpsyc.56.11.1001

92. Cho RY, Konecky RO, Carter CS (2006) Impairments in frontal cortical $\gamma$ synchrony and cognitive control in schizophrenia. Proc Natl Acad Sci USA 103:19878-19883. https://doi.org/10.1073/ pnas.0609440103

93. Spencer KM, Nestor PG, Niznikiewicz MA et al (2003) Abnormal neural synchrony in schizophrenia. J Neurosci 23:74077411. https://doi.org/10.1523/jneurosci.23-19-07407.2003

94. Leicht G, Kirsch V, Giegling I et al (2010) Reduced early auditory evoked gamma-band response in patients with schizophrenia. Biol Psychiatry 67:224-231. https://doi.org/10.1016/j.biops ych.2009.07.033

95. Leicht G, Andreou C, Polomac N et al (2015) Reduced auditory evoked gamma band response and cognitive processing deficits in first episode schizophrenia. World J Biol Psychiatry 16:387397. https://doi.org/10.3109/15622975.2015.1017605

96. Spencer KM, Salisbury DF, Shenton ME, McCarley RW (2008) $\gamma$-Band auditory steady-state responses are impaired in first 
episode psychosis. Biol Psychiatry 64:369-375. https://doi. org/10.1016/j.biopsych.2008.02.021

97. Hong LE, Summerfelt A, McMahon R et al (2004) Evoked gamma band synchronization and the liability for schizophrenia. Schizophrenia Res 2004:293-302

98. Leicht G, Karch S, Karamatskos E et al (2011) Alterations of the early auditory evoked gamma-band response in first-degree relatives of patients with schizophrenia: hints to a new intermediate phenotype. J Psychiatr Res 45:699-705. https://doi. org/10.1016/j.jpsychires.2010.10.002

99. Leicht G, Vauth S, Polomac $\mathrm{N}$ et al (2016) EEG-Informed fMRI reveals a disturbed gamma-band-specific network in subjects at high risk for psychosis. Schizophr Bull 42:239-249. https://doi. org/10.1093/schbul/sbv092

100. Curic S, Leicht G, Thiebes S et al (2019) Reduced auditory evoked gamma-band response and schizophrenia-like clinical symptoms under subanesthetic ketamine. Neuropsychopharmacology 44:1239-1246. https://doi.org/10.1038/s4138 6-019-0328-5

101. Brennan AM, Harris AW, Williams LM (2013) Functional dysconnectivity in schizophrenia and its relationship to neural synchrony. Expert Rev Neurother 13:755-765

102. Mulert C, Kirsch V, Pascual-Marqui R et al (2011) Long-range synchrony of gamma oscillations and auditory hallucination symptoms in schizophrenia. Int J Psychophysiol 79:55-63. https ://doi.org/10.1016/j.ijpsycho.2010.08.004

103. Kornmayer L, Leicht G, Mulert C (2018) Attentional capture by physically salient stimuli in the gamma frequency is associated with schizophrenia symptoms. World J Biol Psychiatry 19:S52S62. https://doi.org/10.1080/15622975.2016.1258491

104. Kornmayer L, Leicht G, Mulert C (2014) Increased gamma oscillations evoked by physically salient distracters are associated with schizotypy. Brain Topogr 28:153-161. https://doi. org/10.1007/s10548-014-0418-y

105. Spencer KM (2012) Baseline gamma power during auditory steady-state stimulation in schizophrenia. Front Hum Neurosci 5:1-7. https://doi.org/10.3389/fnhum.2011.00190

106. Lee KH, Williams LM, Haig A, Gordon E (2003) "Gamma $(40 \mathrm{~Hz})$ phase synchronicity" and symptom dimensions in schizophrenia. Cogn Neuropsychiatry 8:57-71. https://doi. org/10.1080/713752240

107. Gordon E, Williams L, Haig AR et al (2001) Symptom profile and "gamma" processing in schizophrenia. Cogn Neuropsychiatry 6:7-19. https://doi.org/10.1080/13546800042000016

108. Bucci P, Mucci A, Merlotti E et al (2007) Induced gamma activity and event-related coherence in schizophrenia. Clin EEG Neurosci 38:96-104. https://doi.org/10.1177/155005940703800212

109. Lee SH, Wynn JK, Green MF et al (2006) Quantitative EEG and low resolution electromagnetic tomography (LORETA) imaging of patients with persistent auditory hallucinations. Schizophr Res 83:111-119. https://doi.org/10.1016/j.schres.2005.11.025

110. Spencer KM, Nestor PG, Perlmutter R et al (2004) Neural synchrony indexes disordered perception and cognition in schizophrenia. Proc Natl Acad Sci USA 101:17288-17293. https://doi. org/10.1073/pnas.0406074101

111. Baldeweg T, Spence S, Hirsch SR, Gruzelier J (1998) $\gamma$-band electroencephalographic oscillations in a patient with somatic hallucinations. Lancet 352:620-621. https://doi.org/10.1016/ S0140-6736(05)79575-1

112. Grent-t-jong T, Gross J, Goense J et al (2018) Resting-state gamma-band power alterations in schizophrenia reveal e/i-balance abnormalities across illness-stages. Elife 2018:7. https://doi. org/10.7554/eLife.37799

113. Andreou C, Nolte G, Leicht G et al (2015) Increased restingstate gamma-band connectivity in first-episode schizophrenia.
Schizophr Bull 41:930-939. https://doi.org/10.1093/schbul/ sbu121

114. Lee KH, Williams LM, Breakspear M, Gordon E (2003) Synchronous gamma activity: a review and contribution to an integrative neuroscience model of schizophrenia. Brain Res Rev 41:57-78. https://doi.org/10.1016/S0165-0173(02)00220-5

115. Phillips WA, Singer W (1997) In search of common foundations for cortical computation. Behav Brain Sci 20:657-722

116. Bertrand O, Tallon-Baudry C (2000) Oscillatory gamma activity in humans: a possible role for object representation. Int J Psychophysiol 2000:211-223

117. Pettersson-Yeo W, Allen P, Benetti S et al (2011) Dysconnectivity in schizophrenia: where are we now? Neurosci Biobehav Rev 35:1110-1124

118. Li S, Hu N, Zhang W et al (2019) Dysconnectivity of multiple brain networks in schizophrenia: a meta-analysis of resting-state functional connectivity. Front Psychiatry 10:482

119. Stephan KE, Friston KJ, Frith CD (2009) Dysconnection in Schizophrenia: from abnormal synaptic plasticity to failures of self-monitoring. Schizophr Bull 35:509-527

120. Shergill SS, Murray RM, McGuire PK (1998) Auditory hallucinations: a review of psychological treatments. Schizophr Res 32:137-150. https://doi.org/10.1016/S0920-9964(98)00052-8

121. Steinmann S, Leicht G, Andreou C et al (2017) Auditory verbal hallucinations related to altered long-range synchrony of gammaband oscillations. Sci Rep. https://doi.org/10.1038/s41598-01709253-7

122. Mulert C, Kirsch V, Whitford TJ et al (2012) Hearing voices: a role of interhemispheric auditory connectivity? World J Biol Psychiatry 13:153-158. https://doi.org/10.3109/15622 975.2011.570789

123. Steinmann S, Leicht G, Mulert C (2019) The interhemispheric miscommunication theory of auditory verbal hallucinations in schizophrenia. Int J Psychophysiol 145:83-90

124. Meier J, Nolte G, Schneider TR et al (2019) Intrinsic 40Hz-phase asymmetries predict tACS effects during conscious auditory perception. PLoS ONE 14:e0213996. https://doi.org/10.1371/journ al.pone.0213996

125. Sreeraj VS, Shanbhag V, Nawani H et al (2017) Feasibility of online neuromodulation using transcranial alternating current stimulation in schizophrenia. Indian J Psychol Med 39:92-95. https://doi.org/10.4103/0253-7176.198937

126. Sreeraj VS, Shivakumar V, Sowmya S et al (2019) Online theta frequency transcranial alternating current stimulation for cognitive remediation in schizophrenia: a case report and review of literature. J ECT 35:139-143. https://doi.org/10.1097/YCT.00000 00000000523

127. Kallel L, Mondino M, Brunelin J (2016) Effects of theta-rhythm transcranial alternating current stimulation $(4.5 \mathrm{~Hz}$-tACS $)$ in patients with clozapine-resistant negative symptoms of schizophrenia: a case series. J Neural Transm 123:1213-1217. https:// doi.org/10.1007/s00702-016-1574-x

128. Sreeraj VS, Suhas S, Parlikar R et al (2020) Effect of add-on transcranial alternating current stimulation (tACS) on persistent delusions in schizophrenia. Psychiatry Res 290:113106. https:// doi.org/10.1016/j.psychres.2020.113106

129. Hoy KE, Whitty D, Bailey N, Fitzgerald PB (2016) Preliminary investigation of the effects of $\gamma$-tACS on working memory in schizophrenia. J Neural Transm 123:1205-1212. https://doi. org/10.1007/s00702-016-1554-1

130. Hoy KE, Bailey N, Arnold S et al (2015) The effect of $\gamma$-tACS on working memory performance in healthy controls. Brain Cogn 101:51-56. https://doi.org/10.1016/j.bandc.2015.11.002

131. Ahn S, Mellin JM, Alagapan S et al (2019) Targeting reduced neural oscillations in patients with schizophrenia by transcranial 
alternating current stimulation. Neuroimage 186:126-136. https ://doi.org/10.1016/j.neuroimage.2018.10.056

132. Fingelkurts AAA, Fingelkurts AAA (2015) Altered structure of dynamic electroencephalogram oscillatory pattern in major depression. Biol Psychiatry 77:1050-1060

133. Northoff G (2016) How do resting state changes in depression translate into psychopathological symptoms? From 'Spatiotemporal correspondence' to 'Spatiotemporal Psychopathology.' Curr Opin Psychiatry 29:18-24. https://doi.org/10.1097/ YCO.0000000000000222

134. Olbrich S, Arns M (2013) EEG biomarkers in major depressive disorder: discriminative power and prediction of treatment response. Int Rev Psychiatry 25:604-618. https://doi. org/10.3109/09540261.2013.816269

135. Smart OL, Tiruvadi VR, Mayberg HS (2015) Multimodal approaches to define network oscillations in depression. Biol Psychiatry 77:1061-1070. https://doi.org/10.1016/j.biops ych.2015.01.002

136. Leuchter AF, Cook IA, Hunter AM et al (2012) Resting-state quantitative electroencephalography reveals increased neurophysiologic connectivity in depression. PLoS ONE 7:e32508. https://doi.org/10.1371/journal.pone.0032508

137. Olbrich S, Tränkner A, Chittka T et al (2014) Functional connectivity in major depression: Increased phase synchronization between frontal cortical EEG-source estimates. Psychiatry Res Neuroimaging 222:91-99. https://doi.org/10.1016/j.pscychresn s.2014.02.010

138. Vuga M, Fox NA, Cohn JF et al (2006) Long-term stability of frontal electroencephalographic asymmetry in adults with a history of depression and controls. Int J Psychophysiol 59:107-115. https://doi.org/10.1016/j.ijpsycho.2005.02.008

139. Eidelman-Rothman M, Levy J, Feldman R (2016) Alpha oscillations and their impairment in affective and post-traumatic stress disorders. Neurosci Biobehav Rev 68:794-815. https:// doi.org/10.1016/j.neubiorev.2016.07.005

140. Pathak Y, Salami O, Baillet S et al (2016) Longitudinal changes in depressive circuitry in response to neuromodulation therapy. Front Neural Circ. https://doi.org/10.3389/fncir.2016.00050

141. Breitenstein B, Scheuer S, Holsboer F (2014) Are there meaningful biomarkers of treatment response for depression? Drug Discov. Today 19:539-561

142. Keeser D, Karch S, Kirsch V et al (2014) EPA-1603-changes of resting-state eeg and functional connectivity in the sensor and source space of patients with major depression. Eur Psychiatry 29:1. https://doi.org/10.1016/s0924-9338(14)78755-3

143. Iosifescu DV, Greenwald S, Devlin P et al (2009) Frontal EEG predictors of treatment outcome in major depressive disorder. Eur Neuropsychopharmacol 19:772-777. https://doi.org/10.1016/j. euroneuro.2009.06.001

144. Pizzagalli DA, Peccoralo LA, Davidson RJ, Cohen JD (2006) Resting anterior cingulate activity and abnormal responses to errors in subjects with elevated depressive symptoms: a 128-channel EEG study. Hum Brain Mapp 27:185-201. https:// doi.org/10.1002/hbm.20172

145. Fitzgerald PJ, Watson BO (2018) Gamma oscillations as a biomarker for major depression: an emerging topic. Transl Psychiatry 8:1-7. https://doi.org/10.1038/s41398-018-0239-y

146. Strelets VB, Garakh ZV, Novototskii-Vlasov VY (2007) Comparative study of the gamma rhythm in normal conditions, during examination stress, and in patients with first depressive episode. Neurosci Behav Physiol 37:387-394. https://doi.org/10.1007/ s11055-007-0025-4

147. Liu TY, Chen YS, Su TP et al (2014) Abnormal early gamma responses to emotional faces differentiate unipolar from bipolar disorder patients. Biomed Res Int 2014:906104. https://doi. org/10.1155/2014/906104
148. Lee PS, Chen YS, Hsieh JC et al (2010) Distinct neuronal oscillatory responses between patients with bipolar and unipolar disorders: a magnetoencephalographic study. J Affect Disord 123:270-275. https://doi.org/10.1016/j.jad.2009.08.020

149. Isomura $\mathrm{S}$, Onitsuka $\mathrm{T}$, Tsuchimoto $\mathrm{R}$ et al (2016) Differentiation between major depressive disorder and bipolar disorder by auditory steady-state responses. J Affect Disord 190:800-806. https://doi.org/10.1016/j.jad.2015.11.034

150. Alexander ML, Alagapan S, Lugo CE et al (2019) Double-blind, randomized pilot clinical trial targeting alpha oscillations with transcranial alternating current stimulation (tACS) for the treatment of major depressive disorder (MDD). Transl Psychiatry. https://doi.org/10.1038/s41398-019-0439-0

151. Riddle J, Rubinow DR, Frohlich F (2020) A case study of weekly tACS for the treatment of major depressive disorder. Brain Stimul 13:576-577. https://doi.org/10.1016/j.brs.2019.12.016

152. Wilkening A, Kurzeck A, Dechantsreiter E et al (2019) Transcranial alternating current stimulation for the treatment of major depression during pregnancy. Psychiatry Res 279:399-400. https ://doi.org/10.1016/j.psychres.2019.06.009

153. Simpson HB, Tenke CE, Towey JB et al (2000) Symptom provocation alters behavioral ratings and brain electrical activity in obsessive-compulsive disorder: a preliminary study. Psychiatry Res 95:149-155. https://doi.org/10.1016/s0165-1781(00)00177 $-3$

154. Locatelli M, Bellodi L, Grassi B, Scarone S (1996) EEG power modifications in obsessive-compulsive disorder during olfactory stimulation. Biol Psychiatry 39:326-331. https://doi. org/10.1016/0006-3223(95)00172-7

155. Bucci P, Mucci A, Volpe U et al (2004) Executive hypercontrol in obsessive-compulsive disorder: electrophysiological and neuropsychological indices. Clin Neurophysiol 115:1340-1348. https://doi.org/10.1016/j.clinph.2003.12.031

156. Shin YW, Ha TH, Kim SY, Kwon JS (2004) Association between EEG alpha power and visuospatial function in obsessive-compulsive disorder. Psychiatry Clin Neurosci 58:16-20. https://doi.org /10.1111/j.1440-1819.2004.01186.x

157. Pogarell O, Juckel G, Mavrogiorgou P et al (2006) Symptomspecific EEG power correlations in patients with obsessivecompulsive disorder. Int J Psychophysiol 62:87-92. https://doi. org/10.1016/j.ijpsycho.2006.02.002

158. Tot Ş, Özge A, Çömelekoglu Ü et al (2002) Association of QEEG findings with clinical characteristics of OCD: Evidence of left frontotemporal dysfunction. Can J Psychiatry 47:538-545. https ://doi.org/10.1177/070674370204700605

159. Karadağ F, Oğuzhanoğlu NK, Kurt T et al (2003) Quantitative EEG analysis in obsessive compulsive disorder. Int $\mathrm{J}$ Neurosci 113:833-847. https://doi.org/10.1080/00207450390200963

160. Min BK, Kim SJ, Park JY, Park HJ (2011) Prestimulus top-down reflection of obsessive-compulsive disorder in EEG frontal theta and occipital alpha oscillations. Neurosci Lett 496:181-185. https://doi.org/10.1016/j.neulet.2011.04.018

161. Park JY, Lee J, Park HJ et al (2012) Alpha amplitude and phase locking in obsessive-compulsive disorder during working memory. Int J Psychophysiol 83:1-7. https://doi.org/10.1016/j.ijpsy cho.2011.09.014

162. Desarkar P, Sinha VK, Jagadheesan K, Nizamie SH (2007) Subcortical functioning in obsessive-compulsive disorder: an exploratory EEG coherence study. World J Biol Psychiatry 8:196-200. https://doi.org/10.1080/15622970601148547

163. Kamaradova D, Hhajda M, Prasko J et al (2016) Cognitive deficits in patients with obsessive-compulsive disorder-electroencephalography correlates. Neuropsychiatr Dis Treat 12:11191125. https://doi.org/10.2147/NDT.S93040

164. McCarthy PR, Ray WJ, Foa EB (1995) Cognitive influences on electrocortical and heart rate activity in 
obsessive-compulsive disorder. Int J Psychophysiol 19:215-222. https://doi.org/10.1016/0167-8760(95)00009-H

165. Kopřivová J, Congedo M, Horáček J et al (2011) EEG source analysis in obsessive-compulsive disorder. Clin Neurophysiol 122:1735-1743. https://doi.org/10.1016/j.clinph.2011.01.051

166. Clark CR, Galletly CA, Ash DJ et al (2009) Evidence-based medicine evaluation of electrophysiological studies of the anxiety disorders. Clin EEG Neurosci 40:84-112. https://doi. org/10.1177/155005940904000208

167. Velikova S, Locatelli M, Insacco C et al (2010) Dysfunctional brain circuitry in obsessive-compulsive disorder: source and coherence analysis of EEG rhythms. Neuroimage 49:977-983. https://doi.org/10.1016/j.neuroimage.2009.08.015

168. Prichep LS, Mas F, Hollander E et al (1993) Quantitative electroencephalographic subtyping of obsessive-compulsive disorder. Psychiatry Res Neuroimaging 50:25-32. https://doi. org/10.1016/0925-4927(93)90021-9

169. Perera MPN, Bailey NW, Herring SE, Fitzgerald PB (2019) Electrophysiology of obsessive compulsive disorder: a systematic review of the electroencephalographic literature. J Anxiety Disord 62:1-14. https://doi.org/10.1016/j.janxdis.2018.11.001

170. Clementz BA, Sponheim SR, Iacono WG, Beiser M (1994) Resting EEG in first-episode schizophrenia patients, bipolar psychosis patients, and their first-degree relatives. Psychophysiology 31:486-494. https://doi.org/10.1111/j.1469-8986.1994.tb01052.x

171. Özerdem A, Güntekin B, Tunca Z, Başar E (2008) Brain oscillatory responses in patients with bipolar disorder manic episode before and after valproate treatment. Brain Res 1235:98-108. https://doi.org/10.1016/j.brainres.2008.06.101

172. Basar E, Güntekin B, Atagün I et al (2012) Brain's alpha activity is highly reduced in euthymic bipolar disorder patients. Cogn Neurodyn 6:11-20. https://doi.org/10.1007/s11571-011-9172-y

173. El-Badri SM, Ashton CH, Moore PB et al (2001) Electrophysiological and cognitive function in young euthymic patients with bipolar affective disorder. Bipolar Disord 3:79-87. https://doi. org/10.1034/j.1399-5618.2001.030206.x

174. Rommel AS, Kitsune GL, Michelini G et al (2016) Commonalities in EEG spectral power abnormalities between women with ADHD and women with bipolar disorder during rest and cognitive performance. Brain Topogr 29:856-866. https://doi. org/10.1007/s10548-016-0508-0

175. Atagün MI (2016) Brain oscillations in bipolar disorder and lithium-induced changes. Neuropsychiatr Dis Treat 12:589-601. https://doi.org/10.2147/NDT.S100597

176. Kam JWY, Bolbecker AR, O'Donnell BF et al (2013) Resting state EEG power and coherence abnormalities in bipolar disorder and schizophrenia. J Psychiatr Res 47:1893-1901. https://doi. org/10.1016/j.jpsychires.2013.09.009

177. Ethridge LE, Hamm JP, Shapiro JR et al (2012) Neural activations during auditory oddball processing discriminating schizophrenia and psychotic bipolar disorder. Biol Psychiatry 72:766774. https://doi.org/10.1016/j.biopsych.2012.03.034

178. Hamm JP, Ethridge LE, Shapiro JR et al (2012) Spatiotemporal and frequency domain analysis of auditory paired stimuli processing in schizophrenia and bipolar disorder with psychosis. Psychophysiology 49:522-530. https://doi.org/10.111 1/j.1469-8986.2011.01327.x

179. Chen SS, Tu PC, Su TP et al (2008) Impaired frontal synchronization of spontaneous magnetoencephalographic activity in patients with bipolar disorder. Neurosci Lett 445:174-178. https ://doi.org/10.1016/j.neulet.2008.08.080

180. O'Donnell BF, Hetrick WP, Vohs JL et al (2004) Neural synchronization deficits to auditory stimulation in bipolar disorder. NeuroReport 15:1369-1372. https://doi.org/10.1097/01.wnr.00001 27348.64681.b2
181. Özerdem A, Güntekin B, Saatçi E et al (2010) Disturbance in long distance gamma coherence in bipolar disorder. Prog NeuroPsychopharmacol Biol Psychiatry 34:861-865. https://doi. org/10.1016/j.pnpbp.2010.04.001

182. Özerdem A, Güntekin B, Atagün I et al (2011) Reduced long distance gamma $(28-48 \mathrm{~Hz})$ coherence in euthymic patients with bipolar disorder. J Affect Disord 132:325-332. https://doi. org/10.1016/j.jad.2011.02.028

183. Hall MH, Spencer KM, Schulze K et al (2011) The genetic and environmental influences of event-related gamma oscillations on bipolar disorder. Bipolar Disord 13:260-271. https://doi.org/10. 1111/j.1399-5618.2011.00925.x

184. Lima IMM, Peckham AD, Johnson SL (2018) Cognitive deficits in bipolar disorders: implications for emotion. Clin Psychol Rev 59:126-136

185. Dallmer-Zerbe I, Popp F, Lam AP et al (2020) Transcranial alternating current stimulation (tACS) as a tool to modulate P300 amplitude in attention deficit hyperactivity disorder (ADHD): preliminary findings. Brain Topogr. https://doi.org/10.1007/ s10548-020-00752-x

186. Popp F, Dallmer-Zerbe I, Philipsen A et al (2019) Challenges of P300 modulation using transcranial alternating current stimulation (tACS). Front Psychol 10:476. https://doi.org/10.3389/fpsyg .2019.00476

187. Moretti DV, Frisoni GB, Binetti G, Zanetti O (2011) Anatomical substrate and scalp EEG markers are correlated in subjects with cognitive impairment and Alzheimer's Disease. Front Psychiatry 1:152. https://doi.org/10.3389/fpsyt.2010.00152

188. Stam CJ, van Cappellen A, van Walsum AM, Pijnenburg YAL et al (2002) Generalized synchronization of MEG recordings in Alzheimer's disease: evidence for involvement of the gamma band. J Clin Neurophysiol 19:562-574. https://doi. org/10.1097/00004691-200212000-00010

189. de Haan W, Pijnenburg YAL, Strijers RLM et al (2009) Functional neural network analysis in frontotemporal dementia and Alzheimer's disease using EEG and graph theory. BMC Neurosci 10:101. https://doi.org/10.1186/1471-2202-10-101

190. McBride J, Zhao X, Munro N et al (2013) Resting EEG discrimination of early stage alzheimer's disease from normal aging using inter-channel coherence network graphs. Ann Biomed Eng 41:1233-1242. https://doi.org/10.1007/s10439-013-0788-4

191. Babiloni C, Lizio R, Marzano N et al (2016) Brain neural synchronization and functional coupling in Alzheimer's disease as revealed by resting state EEG rhythms. Int J Psychophysiol 103:88-102

192. Rossini PM, Del Percio C, Pasqualetti P et al (2006) Conversion from mild cognitive impairment to Alzheimer's disease is predicted by sources and coherence of brain electroencephalography rhythms. Neuroscience 143:793-803. https://doi.org/10.1016/j. neuroscience.2006.08.049

193. Van Deursen JA, Vuurman EFPM, Verhey FRJ et al (2008) Increased EEG gamma band activity in Alzheimer's disease and mild cognitive impairment. J Neural Transm 115:1301-1311. https://doi.org/10.1007/s00702-008-0083-y

194. Naro A, Corallo F, De Salvo S et al (2016) Promising role of neuromodulation in predicting the progression of mild cognitive impairment to dementia. J Alzheimer's Dis 53:1375-1388. https ://doi.org/10.3233/JAD-160305

195. Demyttenaere K (2019) What is treatment resistance in psychiatry? A "difficult to treat" concept. World Psychiatry 18:354-355. https://doi.org/10.1002/wps.20677

196. Reddy M (2012) Non-compliance in pharmacotherapy. Indian J Psychol Med 34:107-109

197. Mago R (2016) Adverse effects of psychotropic medications: a call to action. Psychiatr Clin N Am 39:361-373 
198. Alagapan S, Schmidt SL, Lefebvre J et al (2016) Modulation of cortical oscillations by low-frequency direct cortical stimulation is state-dependent. PLoS Biol 14:e1002424. https://doi. org/10.1371/journal.pbio.1002424

199. Negrón-Oyarzo I, Aboitiz F, Fuentealba $P$ (2016) Impaired functional connectivity in the prefrontal cortex: a mechanism for chronic stress-induced neuropsychiatric disorders. Neural Plast. https://doi.org/10.1155/2016/7539065

200. Kasten FH, Duecker K, Maack MC et al (2019) Integrating electric field modeling and neuroimaging to explain inter-individual variability of tACS effects. Nat Commun 10:1-11. https://doi. org/10.1038/s41467-019-13417-6

201. Radecke J-O, Andreas K, Carsten H, Schneider TR (2019) Title: simulating individually targeted transcranial electric stimulation for experimental application. bioRxiv 2019:739904. https://doi. org/10.1101/739904

202. Owens E, Bachman P, Glahn DC, Bearden CE (2019) Electrophysiological endophenotypes for schizophrenia. Pharm Rep. https://doi.org/10.1097/HRP.0000000000000110

203. Mulert C, Juckel G, Giegling I et al (2006) A Ser9Gly polymorphism in the dopamine D3 receptor gene (DRD3) and eventrelated P300 potentials. Neuropsychopharmacology 31:13351344. https://doi.org/10.1038/sj.npp.1300984

204. Thut G, Bergmann TO, Fröhlich F et al (2017) Guiding transcranial brain stimulation by EEG/MEG to interact with ongoing brain activity and associated functions: a position paper. Clin Neurophysiol 128:843-857

205. Antal A, Alekseichuk I, Bikson M et al (2017) Low intensity transcranial electric stimulation: safety, ethical, legal regulatory and application guidelines. Clin Neurophysiol 128:1774-1809. https://doi.org/10.1016/j.clinph.2017.06.001

206. Helfrich RF, Schneider TR, Rach S et al (2014) Entrainment of brain oscillations by transcranial alternating current stimulation. Curr Biol 24:333-339. https://doi.org/10.1016/j.cub.2013.12.041

207. Miniussi C, Harris JA, Ruzzoli M (2013) Modelling noninvasive brain stimulation in cognitive neuroscience. Neurosci Biobehav Rev 37:1702-1712. https://doi.org/10.1016/j.neubi orev.2013.06.014

208. Matsumoto H, Ugawa Y (2017) Adverse events of tDCS and tACS: a review. Clin Neurophysiol Pract 2:19-25. https://doi. org/10.1016/j.cnp.2016.12.003

209. Raco V, Bauer R, Olenik M et al (2014) Neurosensory effects of transcranial alternating current stimulation. Brain Stimul 7:823-831. https://doi.org/10.1016/j.brs.2014.08.005

210. Turi Z, Ambrus GG, Janacsek K et al (2013) Both the cutaneous sensation and phosphene perception are modulated in a frequency-specific manner during transcranial alternating current stimulation. Restor Neurol Neurosci 31:275-285. https:// doi.org/10.3233/RNN-120297

211. Laczó B, Antal A, Niebergall R et al (2012) Transcranial alternating stimulation in a high gamma frequency range applied over V1 improves contrast perception but does not modulate spatial attention. Brain Stimul 5:484-491. https://doi.org/10.1016/j. brs.2011.08.008

212. Nekhendzy V, Lemmens HJ, Tingle M et al (2010) The analgesic and antihyperalgesic effects of transcranial electrostimulation with combined direct and alternating current in healthy volunteers. Anesth Analg 111:1301-1307. https://doi.org/10.1213/ ANE.0b013e3181e3697e

213. Fertonani A, Ferrari C, Miniussi C (2015) What do you feel if I apply transcranial electric stimulation? Safety, sensations and secondary induced effects. Clin Neurophysiol 126:2181-2188. https://doi.org/10.1016/j.clinph.2015.03.015

214. Sela T, Kilim A, Lavidor M (2012) Transcranial alternating current stimulation increases risk-taking behavior in the Balloon Analog Risk Task. Front Neurosci 6:22. https://doi.org/10.3389/ fnins.2012.00022

215. Bland NS, Mattingley JB, Sale MV (2018) No evidence for phase-specific effects of $40 \mathrm{~Hz} \mathrm{HD}$-tACS on multiple object tracking. Front Psychol. https://doi.org/10.3389/fpsyg .2018 .00304

216. Noury N, Hipp JF, Siegel M (2016) Physiological processes nonlinearly affect electrophysiological recordings during transcranial electric stimulation. Neuroimage 140:99-109. https://doi. org/10.1016/j.neuroimage.2016.03.065

217. Noury N, Siegel M (2017) Phase properties of transcranial electrical stimulation artifacts in electrophysiological recordings. Neuroimage 158:406-416. https://doi.org/10.1016/j.neuroimage .2017.07.010

218. Asamoah B, Khatoun A, Mc Laughlin M (2019) tACS motor system effects can be caused by transcutaneous stimulation of peripheral nerves. Nat Commun 10:1-16. https://doi. org/10.1038/s41467-018-08183-w

219. Lafon B, Henin S, Huang Y et al (2017) Low frequency transcranial electrical stimulation does not entrain sleep rhythms measured by human intracranial recordings. Nat Commun. https ://doi.org/10.1038/s41467-017-01045-x

220. Schutter DJLG, Hortensius R (2010) Retinal origin of phosphenes to transcranial alternating current stimulation. Clin Neurophysiol 121:1080-1084. https://doi.org/10.1016/j.clinp h.2009.10.038

221. Schutter DJLG (2016) Cutaneous retinal activation and neural entrainment in transcranial alternating current stimulation: a systematic review. Neuroimage 140:83-88. https://doi. org/10.1016/j.neuroimage.2015.09.067

222. Karabanov AN, Saturnino GB, Thielscher A, Siebner HR (2019) Can transcranial electrical stimulation localize brain function? Front Psychol 10:213

223. Feusner JD, Madsen S, Moody TD et al (2012) Effects of cranial electrotherapy stimulation on resting state brain activity. Brain Behav 2:211-220. https://doi.org/10.1002/brb3.45

224. Guleyupoglu B, Schestatsky P, Edwards D et al (2013) Classification of methods in transcranial Electrical Stimulation (tES) and evolving strategy from historical approaches to contemporary innovations. J Neurosci Methods 219:297-311

225. Vieira PG, Krause MR, Pack CC (2020) tACS entrains neural activity while somatosensory input is blocked. bioRxiv 2020:691022. https://doi.org/10.1101/691022

226. Fiene M, Schwab BC, Misselhorn J et al (2020) Phase-specific manipulation of rhythmic brain activity by transcranial alternating current stimulation. Brain Stimul 13:1254-1262. https://doi. org/10.1016/j.brs.2020.06.008 